\title{
ANALYTICAL STUDY OF A CONTROL ALGORITHM BASED ON EMOTIONAL PROCESSING
}

\author{
A Thesis \\ by \\ MANIK CHANDRA \\ Submitted to the Office of Graduate Studies of \\ Texas A\&M University \\ in partial fulfillment of the requirements for the degree of \\ MASTER OF SCIENCE
}

December 2005

Major Subject: Mechanical Engineering 


\title{
ANALYTICAL STUDY OF A CONTROL ALGORITHM BASED ON EMOTIONAL PROCESSING
}

\author{
A Thesis \\ by \\ MANIK CHANDRA \\ Submitted to the Office of Graduate Studies of \\ Texas A\&M University \\ in partial fulfillment of the requirements for the degree of \\ MASTER OF SCIENCE
}

Approved by:

Chair of Committee, Reza Langari Committee Members, Luis San Andres John Valasek Head of Department Dennis L. O’Neal

December 2005

Major Subject: Mechanical Engineering 


\begin{abstract}
Analytical Study of a Control Algorithm Based on Emotional Processing. (December 2005)

Manik Chandra, B. Tech., Indian Institute of Technology Kanpur, India Chair of Advisory Committee: Dr. Reza Langari
\end{abstract}

This work presents a control algorithm developed from the mammalian emotional processing network. Emotions are processed by the limbic system in the mammalian brain. This system consists of several components that carry out different tasks. The system level understanding of the limbic system has been previously captured in a discrete event computational model. This computational model was modified suitably to be used as a feedback mechanism to regulate the output of a continuous-time first order plant. An extension to a class of nonlinear plants is also discussed.

The combined system of the modified model and the linear plant are represented as a set of bilinear differential equations valid in a half space of the 3-dimensional real space. The bounding plane of this half space is the zero level of the square of the plant output. This system of equations possesses a continuous set of equilibrium points which lies on the bounding plane of the half space.

The occurrence of a connected equilibrium set is uncommon in control engineering, and to prove stability for such cases one needs an extended Lyapunov-like theorem, namely 
LaSalle's Invariance Principle. In the process of using this Principle, it is shown that this set of equations possesses a first integral as well. A first integral is identified using the compatibility method, and this first integral is utilized to prove asymptotic stability for a region of the connect equilibrium set. 
To all impediments and distractions 


\section{ACKNOWLEDGMENTS}

I am incapable of describing my gratitude to my parents and my brother. Without their

influence I would not be the same person that I am and this work could never have materialized.

I would also take this opportunity to thank my advisor, Dr Langari, for patiently bearing my shortcomings and helping me identify my strengths as I inched ahead through this work. I am grateful to him for setting high standards and allowing me the time to reach them. 


\section{TABLE OF CONTENTS}

CHAPTER $\quad$ Page

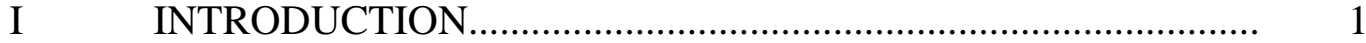

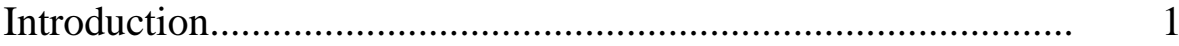

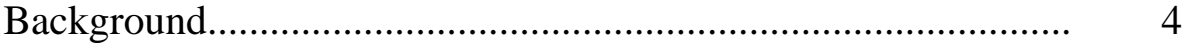

Overview of the thesis.............................................................. 9

II COMPUTATIONAL MODELING OF THE EMOTIONAL

PROCESSING NETWORK....................................................... 10

The limbic system............................................................... 10

The architecture of the limbic system..................................... 12

A computational model of the limbic system............................ 15

Previous work....................................................................... $\quad 20$

Application to a linear system............................................. 21

III $\quad$ EQUILIBRIUM SETS, INVARIANT SETS AND STABILITY... 26

A qualitative understanding of the system................................. 26

Few definitions..................................................................... 28

Stability in the presence of equilibrium sets............................. $\quad 30$

IV FIRST INTEGRALS OF DIFFERENTIAL EQUATIONS........... 32

What are first integrals?......................................................... 32

Need of first integrals............................................................ 33

A method to find first integrals.................................................. 34

Finding a first integral to our problem.................................... 37

V PROOF OF STABILITY ........................................................ 43

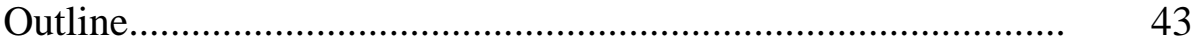

A 2-dimensional example.......................................................... 44

Construction of positively invariant sets for the complete

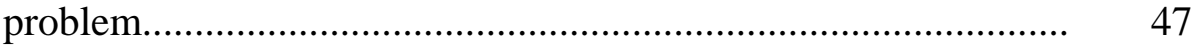

Main proof............................................................................. 51

VI CONCLUSION

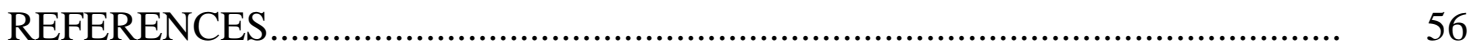

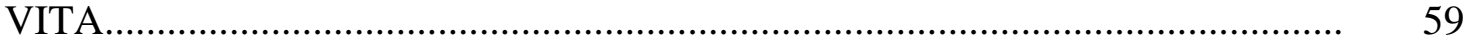




\section{LIST OF FIGURES}

FIGURE Page

1 Feedback error learning scheme to acquire inverse model of the controlled object.......................................................... 6

2 A generic view of the important components of the human limbic system.................................................................. 12

3 A computational model of the limbic system....................... 16

$4 \quad$ The plant in a closed loop with the controller........................... 21

5 The construction of positively invariant sets in 2-dimensional problem........................................................................ 46

6 The nature of flow represented in any plane $x_{1}=$ positive constant................................................................................. 48

$7 \quad$ Construction of compact positively invariant sets in the various regions demarcated by the nullcline planes in the 3dimensional problem............................................................... 49

$8 \quad$ Construction of compact positively invariant sets by union...... 52 


\section{CHAPTER I \\ INTRODUCTION}

\section{Introduction}

Increased scientific attention is being drawn towards understanding the decision architecture of the Human Brain. Interest has been showed by neurophysiologists, cognitive scientists, artificial intelligence researchers, and control engineers. Till late, emotions were considered a negative trait by neurophysiologists and cognitive scientists and were considered a weakness of the human individual. Rational thought is considered to be objective and repetitive [1]. Repetition suggests the existence of a well defined mapping between input and outputs that shall be followed by the system and multiple instances would allow the decision system to reveal this hidden relation.

On the other hand, Emotional thought is considered to be involuntary and there exists little conscious control over such thought. Nonetheless, these involuntary emotions can easily and frequently change conscious thought [1]. Thus under certain conditions, inner symptoms and cues are given more relevance than external stimuli. LeDoux [2] presented several examples where the emotional capability of a human subject was lost due to some accident/surgery; however cognition and other capabilities were unaffected. This indicates that the two systems, the emotional system and the cognitive system, are separate and also that the connection from the emotional to the cognitive system is far stronger than the reverse relation.

This thesis follows the style of IEEE Trans. of Systems, Man and Cybernetics. 
However it has been understood lately that emotions actually help in learning. As an example [3]: one single occurrence of an emotionally charged-up situation remains in one's memory for years to come, whereas the memory of some task that one performs daily would not be equally vivid and easily recalled. This actually questions the common perception that repetition 'memorizes' information into our brain. Through this example, one can also comment that sustained cognitive inputs were not able to develop the effect that one single internal cue can generate.

Learning is a process by which a decision scheme's outputs are made more appropriate based upon experience. An appropriate decision is one which makes the attainment of the desired goal more probable [4]. Hence research in artificial intelligence is also aiming to understand the value judgments that emotions bring in. Situations such as an unmanned vehicle in a battle scenario need an artificial driver which would be able to emulate a human's decision mechanism and take the right decision in "real-time" and also incorporate information from previous exposures to similar scenarios. In fact, Samad mentions that the only intelligent systems that exist are biological and hence biomimicry is the first logical step to develop artificial intelligence and autonomous systems, unless one can think about and develop an autonomous system which is not inspired by a biological system [5]. Even the development of a non-biologically inspired autonomous system would involve the study of biological intelligence and search for its shortcomings. 
The emotional system has also been attributed the task of what engineers would call multi-objective optimization. Without emotions, a human being would not be able to develop complicated ideas and thoughts. Without the emotional system, a human brain would only process and evaluate different stimuli for different objectives: the interrelated evaluation and associations, which we call as context, are also provided by the emotional system. For example: compromises and strategies would not be possible without the relative emotional prioritization of various goals [1]. In fact this very prioritization demarcates species of higher intelligence from those of lower intelligence. Young reptiles have to fend for their own food and can be eaten by their own mothers, while primates are able to make compromises and provide nutrition to their young ones.

The evaluation or prioritization that each individual allocates to the same set of stimuli, introduces individual traits. A particular individual may allocate more importance to a particular feature or emotion so that its decision would be strikingly different from another individual's. Again the outcomes of such previous situations would ensure the evaluations that the particular individual would make in the current situation. Note that such emotional prioritization in individuals is at a level higher than the varying ability of emotional prioritization in different species as shown in the example above. Albus [4] terms such individual prioritization as derived from the higher levels of the so-called Behavior Generating Hierarchy. 


\section{Background}

From a control engineering perspective, the task of learning and making decisions more appropriate is the purview of adaptive control. By bio-inspired adaptive control, one's attention is readily drawn towards Artificial Neural Networks. However, artificial neural networks model the synaptic connections and the Hebbian learning phenomena at the level of individual neurons, which allow incorporating complex information into the decision scheme where no conventional or mathematical input-output relations are available. One may also consider emotions to be intangible and un-modeled; and eventually, inside the human brain, they are represented in networks of neurons. We will explore, in a later paragraph that Artificial Neural Networks are relevant and are being used for some studies, but the habituation or sensitization of neurons is at a much lower level than the emotional mechanism. The brain emotional mechanism is a macro-level setup which would incorporate a very large number of neurons clustered into various components of the emotional system network. By looking at the emotional system, one is looking at and emulating the system level architecture that a biological intelligence scheme would have. One need not choose an artificial network of neurons to implement this system level design.

Albus and Samad, as referred earlier, have individually proposed that the most logical and probable strategy towards understanding and implementing artificial intelligence is to understand and mimic the biological decision architecture present in the central nervous system of humans and other higher mammals. This higher mammalian central 
nervous system has been perfected over millions of years of evolution and is very much the standard and de facto decision scheme against which our entire understanding of basic concepts of intelligence, learning and autonomy is defined and verified.

Interestingly there exists a reverse inclination amongst a school of neurophysiologists who believe that the easiest way to understand the complicated functionalities of the brain and the central nervous system is to model them as adaptive control models, or as their artificial neural network equivalents [6], [7].

The main interest that neurophysiologists had was to identify how the brain was able to handle tasks such as involuntary bodily processes, reflex action as well as voluntary motor coordination, cognition, etc. And why different parts of the brain were utilized for such purposes, as was experimentally discovered through innumerable electroencephalogram experiments on normal and special individuals. The main agenda was to identify modes in which the central nervous system's components behaved. For example [7]: classifying the task as of adaptive signal processing or adaptive filtering. In fact, a more structural approach also exists which classifies the components acting as a scaling device, (which will be called a gain in control engineering terminology) vis-à-vis a time-delay device (a differentiator/integrator).

Due to the complexity of the mammalian nervous system, this study is not limited to theorizing models [6] but intricate experiments [7] have also been used to validate these 
models. And this, in a way, lends force to the earlier proposition that a clear understanding of natural intelligence is a logical step towards modeling intelligence and autonomy into artifacts.

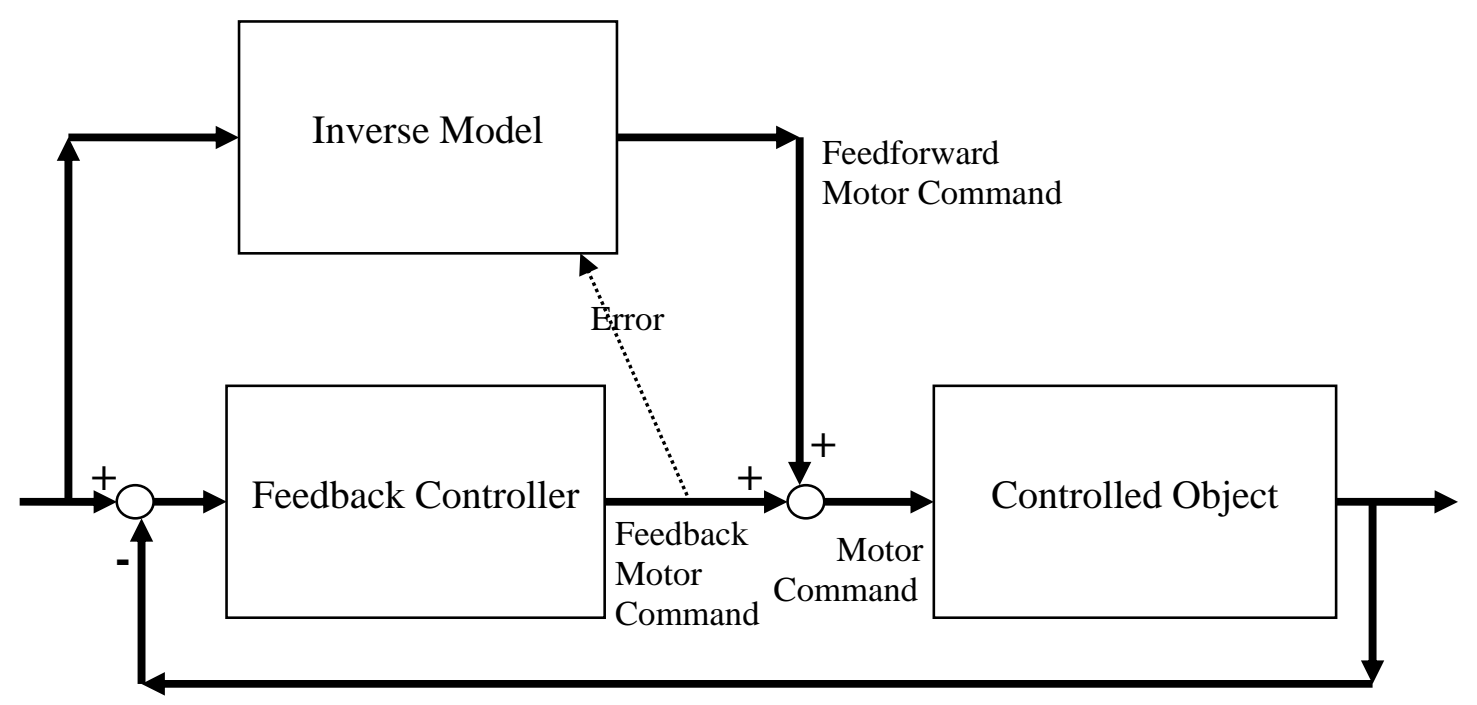

Figure 1: Feedback error learning scheme to acquire inverse model of the controlled object (as referenced in [7]).

Barlow [7] presents a number of control models, developed by several researchers of this school of thought, to explain the architecture of the human brain, specifically the human cerebellum. Barlow summarizes the various attempts by several researchers over decades, and shows that the tendency is towards an adaptive control representation, due to the several features that Adaptive Control Theory offers, e.g. time-development of parameters, even under situations where limited prior knowledge of the system is available. Earlier non-adaptive models were proposed, but they suffered from several shortcomings which only adaptive systems could provide. Figure 1 [7] presents a schematic flow diagram of motor behavior acquisition in vertebrates. Any control 
engineer would clearly identify this diagram as the closed loop diagram of an adaptive process. Barlow also presents a number of Artificial Neural Network models of the cerebellum and other parts of the brain involved in complete circuits of some bodily task. It is also pointed that the use of Artificial Neural Networks is also due to its ability to manipulate weights of the various signal paths according to the error between the desired state and the current state.

However the models presented therein are mainly explanations of how the human cerebellum acts like an adaptive controller while regulating bodily processes like posture and locomotion. A control mechanism based on the emotional setup in the brain is still not available.

Apart from the role that emotions play, the human brain is known to be having several redundant connections in its setup. In fact calling them redundancies is not very appropriate as these alternate pathways provide robustness and also validate and support each other's outputs. Also all connections are not identical, some of them are fast connections, and often inferior in the quality of judgment they result in. The Limbic System, which, due to experimental work by neurophysiologists, is considered to be the emotional processing mechanism in the mammalian nervous system, is made of two loops. The Orbitofrontal Cortex acts as an outer loop and receives information from the Amygdala, as well, which only receives sensory input. It is interesting to note that emotional responses can occur without the sensor information going into the higher 
brain, where more complicated processing is undertaken [2]. Hence this loop structure provides for a mechanism where a quick decision from the inner loop can be actually modified by the outer loop.

Hence an emotion-based control mechanism has still not been researched primarily due to two reasons: the role of emotions in the human brain was not understood and also due to the unavailability of a mathematical/computational model of the emotional learning mechanisms in the brain.

However it should be clear from the outset that by an emotion based control mechanism, one does not mean an artificial emotion processing setup or an artificial agent that can generate emotions. Neither are we attempting an Artificial Intelligence problem. Artificial Intelligence and control systems have different goals. In fact, by itself, emotional learning and intelligence are not equivalent: emotional learning is only a small but crucial component of the entire concept of intelligence. Besides emotions, an intelligent being ought to display perception, reasoning, planning, self-consciousness besides other high level abstract features which have little to do with control engineering [3], [4]. 


\section{Overview of the thesis}

After having introduced and discussed the background necessary for this study in the preceding pages, we now give the outline of the document here. The next chapter discusses the core parts of the mammalian emotional network, which is called the Limbic System. It also discusses a computational model which was derived for cognitive studies, which is then suitably modified for application to a linear system. Chapter III discusses the unusual equilibrium nature of the system developed in chapter II and provides some definitions from stability theory and discusses the issues that need to be addressed for proving stability. Then in chapter IV we present the concept of first integrals related to differential equations which are of benefit to our task of proving stability. Finally in chapter $\mathrm{V}$ an outline of a proof of stability of the system of ordinary differential equations is presented. And we conclude in chapter VI with a few remarks about the present developments and their limitations and probable future work. 


\section{CHAPTER II}

\section{COMPUTATIONAL MODELING OF THE EMOTIONAL}

\section{PROCESSING NETWORK}

\section{The limbic system}

The emotional processing setup inside the mammalian brain is called the limbic system. The limbic system is closely associated with the functions of memory and learning, besides emotional processing and emotional learning. The limbic system evolved further into what is called the neocortex configuration [1]. The neocortex configuration was the system that allowed Homo sapiens to have an emotional life, including complicated combinations of elementary and even contradictory emotions. The size of the neocortex is considered proportional to the complexity and the breadth of the spectrum of the emotions the individual feels (or more correctly 'emotes'). This occurs due to the existence of more neuron connections within the limbic system or the neocortex, which allow the processing of multiple and more comprehensive responses to the same set of stimuli. It is the occurrence of these varied responses in higher animals that marks them as intelligent and 'higher' than other animals whose central nervous systems lacks the limbic setup and hence can only process the inputs to fewer and often single responses. E.g. when threatened a lower animal will flee, whereas higher animals might behave along different plans. 
The name limbic system was suggested by MacLean in 1952 [2] to a regrouping of a part called limbic cortex with other parts. The term 'limbic' in the limbic cortex had a structural implication, whereas MacLean's sense was more function-oriented when he clubbed other related sub cortical regions to form the system. When this theory was put forth, the experimental sciences were not as well developed, so MacLean suggested that the primitive architecture of this setup leads to the irrationality, multiplicity and confusion about emotional behavior. However later on it emerged that the existence of several well-defined loops or processing paths actually provide this setup a sophisticated computational power which accounts for complicated emotional behavior.

It should be clarified here that the current understanding of the limbic system and the neocortex was never static and has been disputed often and changes suggested periodically. LeDoux [2] in his book gives a good discussion about it. MacLean's theory about the limbic system has not been able to generate sufficient experimental evidence; however some parts of this theory have not been discarded outright and have been accepted as being true though poorly organized. Hence usually a consensus is agreed upon which says that some limbic areas of the brain are related with certain emotional processes and such relations are generalized to say that the entire limbic system is related to emotional thought and processing. LeDoux goes further to even say that the classical limbic areas have been experimentally shown to not being dedicated to emotions, but the theory still persists, as an elementary proposition that links evolution and emotions. However, our emphasis here is engineering and for our engineering goals, 
such a theory is good enough to pick up and try a few ideas that might not have been studied as yet and can lend important insight into engineering as well as the original biological context.

\section{The architecture of the limbic system}

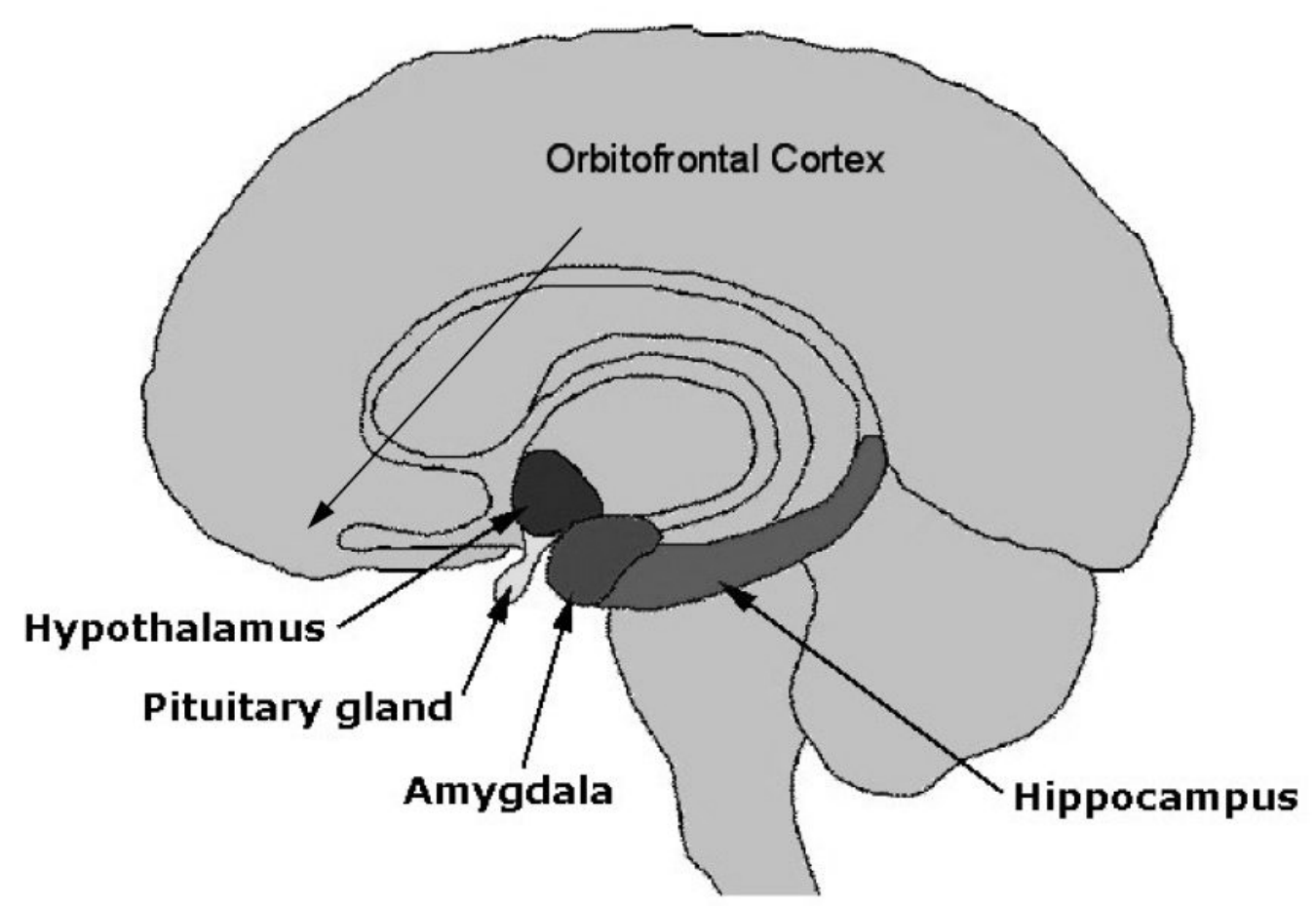

Figure 2: A generic view of the important components of the human limbic system. Other higher mammals also possess similar structure.

The primary components of the human limbic system are shown above in Figure 2. The amygdala and the orbitofrontal cortex are the main components involved in emotional processing. Amygdala is the lobe where the stimuli from the sensory lobes are mapped to emotional responses [8]. It has been experimentally verified that this lobe undergoes 
classical conditioning over the various stimuli presented to it. The output of this lobe is further transmitted to hypothalamus and other structures. The hypothalamus is responsible for generating the emotional reactions. The amygdala receives three kinds of input signals: first, the sensory information from the sense organs; second, the internal significance of the stimuli, and third, the mode of operation for the being itself. [8] As can be seen these signals mix external and internal cues to deliver complete information and context to the individual.

The orbitofrontal cortex has been experimentally shown to possess an inhibitory role in the whole emotional processing network. The orbitofrontal cortex tries to reduce the strength of earlier established connections which are no longer appropriate as the goal or the context has changed. Hence one can say that the orbitofrontal cortex is responsible for the working memory of the individual which comprises of similar events in the past and a representation of the events and actions of the present situation. Hence the orbitofrontal cortex regulates the mapping of the stimuli to the emotional response occurring through the amygdala. This can be called habituation of the senses to some stimulus which though present repeatedly has little relevance to the main mode of operation or has delivered its information content to the brain and has therefore lost its importance status, and the focus has shifted after assimilating this information content. The Hippocampus is believed to provide the orbitofrontal cortex with information about the current context. The orbitofrontal cortex receives the same set of data as the amygdala, but it forms a sort of outer loop as it also receives the amygdala states. 
Hence the orbitofrontal cortex tracks the difference between the system predictions and the actual reinforcer from the amygdala and learns to inhibit the system output proportionate to the mismatch. Different regions of the orbitofrontal cortex are functionally related to the different sensory stimuli and provide different levels of regulatory action to different stimuli.

The thalamus acts as a relay organ between the cortical parts and the other parts of the network. It is believed that the thalamus alters the sensory stimuli with some emotionally charged stimuli. However this mechanism is not very clearly understood as yet [8]. For instance the olfactory stimuli do not pass through the thalamus.

The hypothalamus is situated below the thalamus and mainly operates on the endocrine system and the pituitary gland. The pituitary gland is the all important center of the endocrine system where hormonal secretion is controlled which enacts through the different body organs, all the emotional processing undertaken by the limbic system.

As was pointed earlier, the understanding of the limbic system is not accurate and equally convincing in all regards. Hence neurophysiologists are still actively engaged in refining and suggesting newer explanations of the emotional processing system. A keen reader with a background in neuroanatomy can find several other published resources similar to [2], [8], [9], [10]. 


\section{A computational model of the limbic system}

From an engineering viewpoint we need a computational model of the emotional processing network that can represent the system relationships of the individual elements. What the previous pages discussed was a descriptive model of the emotional processing network. It was a qualitative discussion about the various parts of the brain and more stress was given to make the functioning of the system easy to understand. Such a model presents the salient features only and serves well for an introduction. However little stress was laid upon how this understanding was attained or developed and one can easily be mislead with a model that appears to explain the underlying phenomena, but is flawed or may not be verified as exhaustively as it should be.

By a computational model, one implies a model which can be worked with. It is not a description of the phenomena, but is rather an attempt to show it running. A computational model through this definition appears to be a model that can be represented as a set of equations that quantitatively define the phenomenon. From an engineering viewpoint, a computational model is the outcome of a system identification process: not only is the functional form identified but also the parameter values are also learnt. For the current aim of picking up the limbic system and using it for control engineering purposes, there is no need to identify the parameter values. The parameters would be set by the control engineering problem that one would like to study using this emotional processing model. 
Morén and Balkenius [11], and Morén's Ph.D. thesis [8] offer us a computational model of the limbic system. This model is certainly not complete and neither does it intend to equally capture all dimensions of the human limbic architecture. However it has been verified by the authors above to provide simulation results which match with experimental data and also agree with the descriptive understanding developed in the field of brain modeling. Hence such a model agrees with our definition of the computational model, that we just elucidated above.

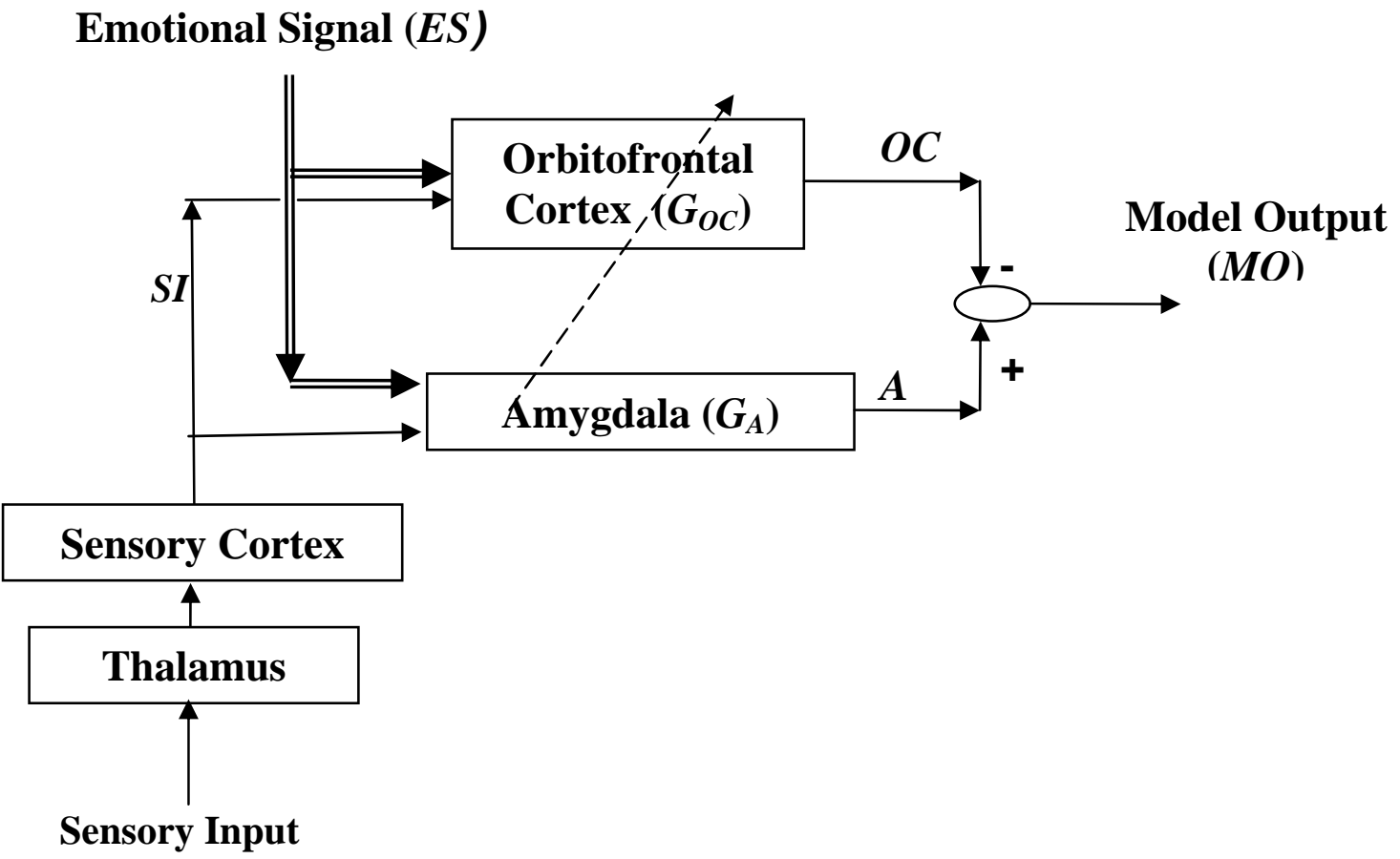

Figure 3: A computational model of the limbic system [8].

Figure 3 above represents the Morén-Balkenius model in a simple, yet effective manner and which is good enough for our engineering aims. Primarily it can be seen that there are two input signals: namely the sensory input coming from the stimulus being 
registered in the sense organs. The other signal called the Primary Reward is an internal signal. In fact, the understanding of this Primary Reward signal (also referred to as Emotional Signal ES, for reasons to be mentioned later) is inconclusive as yet: the exact source of this Emotional Signal ES is unclear, as of now it is considered to be a generic signal from the thalamus, hypothalamus and the basal ganglia regions of the brain. The understanding of the main parts of the emotional processing network has not been mastered, as yet; hence our understanding of these regions is all the more primitive. In fact this lack of previous information is a good sign for innovation, and the generation of the reward signal will become important for our study within the next few pages.

The functional relationships between the various signals are now defined. The signal entering the orbitofrontal cortex (OFC) and the amygdala blocks are called the $S I_{i}$ for Sensory Input, as processed (or filtered) through the sensory cortex and the thalamus. Note that here all quantities have a subscript, as inside the natural emotional processing network, there are several such loops, and all of the outputs from individual loops are summed up in the end to give the Model Output MO. The amygdala and the OFC blocks are basically gains, $G_{A_{i}}$ and $G_{O C_{i}}$ acting over the Sensory Input $S I$ and these gains are updated by $\Delta G_{A_{i}}$ and $\Delta G_{O C_{i}}$ depending on the emotional signal and other signals. The output signal from the amygdala is $A_{i}$ and that from the orbitofrontal cortex is the $O C_{i}$. The following lists the signal representations and the update rules that actually define the mathematics inside the emotional processing network, as given in [8]: 


$$
\begin{aligned}
A_{i} & =G_{A_{i}} \cdot S I_{i} \\
O C_{i} & =G_{O C_{i}} \cdot S I_{i} \\
\Delta G_{A_{i}} & =\alpha \cdot S I_{i} \cdot \max \left(0, E S-\sum_{i} A_{i}\right) \\
\Delta G_{O C_{i}} & =\beta \cdot S I_{i} \cdot R_{o} \\
\text { where } R_{0} & =\left\{\begin{array}{cc}
\max \left(0, \sum_{i} A_{i}-E S\right)-\sum_{i} O C_{i} & \forall E S \neq 0 \\
\max \left(0, \sum_{i} A_{i}-\sum_{i} O C_{i}\right) & \forall E S=0
\end{array}\right\} \\
M O & =\sum_{i} A_{i}-\sum_{i} O C_{i}
\end{aligned}
$$

Here $\alpha, \beta$ are constants and represent rates of learning for the individual components. It should be noted that $\alpha$ and $\beta$ remain the same over the various loops. A higher value of $\alpha, \beta$ means that the system values are updated faster, as in each iterative pass, the update is larger. $\mathrm{R}_{\mathrm{o}}$ is called the internal reinforcer for the orbitofrontal cortex (OFC).

Also note that the manner in which these equations are constructed, the updates or the learning in the amygdala gain $G_{A_{i}}$ are monotonic: the amygdala gain $G_{A_{i}}$ can only increase. However the OFC gain update rules are not monotonic. In the presence of a reward, the internal reinforcer represents the discrepancy between the reward and the amygdala outputs $A_{i}$ minus the OFC output $O C_{i}$. However if there is no reward, then the OFC behaves differently: the internal reinforcer is only the surplus of the amygdala outputs over the OFC outputs $\left(O C_{i}\right)$. That means that without a reward, the OFC gain $G_{O C i}$ is updated upwards if the amygdala responds more sharply than the OFC. Hence this leads to a setup in which OFC regulates the amygdala response. An interpretation can be worked out that the amygdala attempts to match the reward/emotional signal and 
whenever it is unable to do so the orbitofrontal cortex (OFC) tries to fine-tune the system. Hence it can be said that the amygdala tries to learn the associations between the sensory and the emotional inputs. And the updates of amygdala are monitored by the OFC gains, which inhibit the amygdala if the response is more acute than necessary. Note that as the amygdala gain updates are monotonic, the amygdala gains are not inhibited, but the amygdala response is inhibited by increasing the OFC gain.

The set of rules as defined by these equations is independent of time as of now. Morén [8] comments that the absence of time related effects is the shortcoming of this model, and all stimuli are considered to be pulses, lasting one clock period.

One also needs to identify the Emotional Signal ES or the Primary Reward. Morén [8] outlines several theories for the Reward or the Reinforcing Signal. The Emotional Signal is of importance to the entire model. It is understood that this signal is generated internally \& combines external sensory inputs with internally generated cues to develop a complete understanding of the situation: internal as well as external. The internal cues represent the goals of the individual against which it is comparing its performance as measured by the sensory inputs. Hence this signal is called the Emotional Signal. In fact if we pay attention to LeDoux's definition [2] quoted below then it is more appropriate to call this signal as emotional signal, which is what we will follow in this thesis.

"When information from the external world is integrated with sensations arising from within the body, we have feelings." 


\section{Previous work}

A number of attempts have been reported [12], [13] which utilize the above model for varied control engineering applications: e.g. control of MIMO and nonlinear systems. These articles used the Morén-Balkenius model as a feedback law to generate control input to the plants. However no reasoning into the probable causes for the applicability of this model for such tasks was presented. One should not implement any function as a feedback law: firstly, an underlying rationale for doing so should be available and secondly a method should be available in order to generalize the concepts introduced so that a controller can be designed for other problems as well.

Also the examples presented were not in continuous time mode: these were Simulink ${ }^{\circledR}$ based simulations of continuous time descriptions of plants being fed back with a control scheme which was in discrete time mode. The gain development rules were written as difference equations, very close to what Morén presented.

However these results present an initial interest in the area of using ideas from the emotional processing network for control engineering purpose. Interest in understanding biological systems and modifying salient features for application to artificial systems is the most prominent thrust in engineering research as of now. They have presented that the emotional processing network is a suitable candidate for feedback law and more work is needed to understand it. 


\section{Application to a linear system}

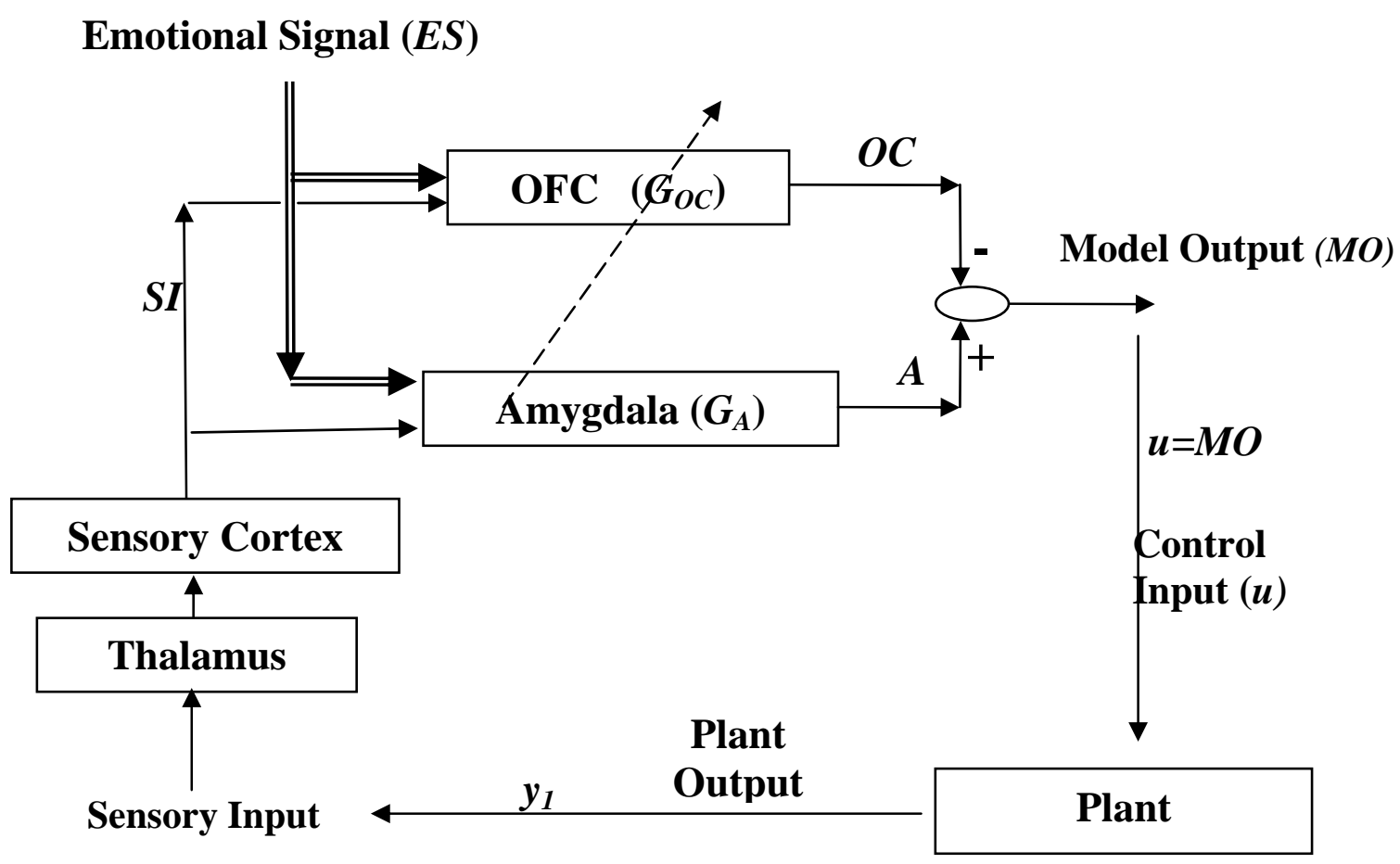

Figure 4: The plant in a closed loop with the controller.

The Morén-Balkenius model is now adapted to be used as the feedback law for the regulation of a first order linear time invariant system given by:

$$
\dot{y}_{1}=-a y_{1}+b u
$$

where $y_{1}$ is the state, $u$ is the control input and $a>0$. Figure 4 outlines this feedback law. Using the limbic system model, we propose the following linear feedback rule which uses the model output $M O$ for a single set of amygdala and OFC (i.e. $i=1$ only)

$$
M O=A_{1}-O C_{1}=\left(G_{A^{-}} G_{O C}\right) \cdot S I
$$

as the control input $u$ for the system. As the amygdala and the OFC gains are dynamic in nature, we define two more state variables, $y_{2}$ and $y_{3}$ representing $G_{A}$ and $G_{O C}$ 
respectively. We also define the Sensory Input SI to be the plant output or state $y_{1}$. Note that we are allowed flexibility in deciding the $S I$ and it can involve derivatives of the plant output as well, but for analytical simplicity we have chosen $S I$ to be $y_{1}$. Hence we can write the following:

$$
u=\left(y_{2}-y_{3}\right) y_{1}
$$

Now we define the Reward or the Emotional Signal ES as a combination of the external signal $x_{1}$ (external to the controller) and the internal signal $u$.

$$
E S=w_{1} \cdot y_{1}+w_{2} \cdot u
$$

The weights $w_{1}$ and $w_{2}$ help in defining the relative importance that has to be given to either signal. Actually the external signal should be the error, but as this is a regulation problem, the error is identical to the output. From a biological perspective, by mixing these two signals we are attempting to incorporate the separate information of what needs to be done and what can be done at the present situation.

The update rules are rewritten to relate the time derivatives of the gains with the same functional form as in the emotional processing network. This is valid as it can be understood that the discrete updates are occurring at a high frequency and the sampling time can be absorbed into the constants. Also we remove the max operator as well, for analytical simplicity. Further work shall show that for continuous time development the maximum operator is not required as such. 
Hence (1) is simplified to yield the following set of equations:

$$
\begin{aligned}
A & =G_{A} \cdot S I \\
O C & =G_{O C} \cdot S I \\
\frac{d G_{A}}{d t} & =\alpha \cdot S I .(E S-A) \\
\frac{d G_{O C}}{d t} & =\beta \cdot S I .(A-O C-E S) \\
M O & =A-O C
\end{aligned}
$$

The above equations capture the supervisory nature of the orbitofrontal cortex (OFC) in an elementary sense. In the presence of a nontrivial Sensory Input SI, if one of the gains $G_{A}$ or $G_{O C}$ stops evolving, i.e. when the Emotional Signal ES equals the amygdala output A or when the Model Output MO equals the Emotional Signal ES, then the update of the other gain depends on the magnitude of the OFC output $O C$.

Hence after incorporating (4) and (5) we write the update rules as:

$$
\begin{aligned}
& \dot{y}_{2}=\alpha\left(\left(w_{2}-1\right) y_{2}-w_{2} y_{3}+w_{1}\right) y_{1}^{2} \\
& \dot{y}_{3}=\beta\left(\left(1-w_{2}\right) y_{2}-\left(1-w_{2}\right) y_{3}-w_{1}\right) y_{1}^{2}
\end{aligned}
$$

Hence (2), (4), (7) and (8) make up the closed loop system. We can see that the feedback law is linear in terms of the plant output but the gains are time varying and these variations are nonlinear in plant output.

For further analytical simplicity if we define $x_{1}$ as $y_{1}^{2}, x_{2}$ as $\left(y_{2}-y_{3}\right)$ and $x_{3}$ as $y_{3}$ then the above system of equations can be written as:

$$
\frac{d}{d t}\left[\begin{array}{l}
x_{1} \\
x_{2} \\
x_{3}
\end{array}\right]=\left[\begin{array}{c}
2\left(b x_{2}-a\right) \\
(\alpha+\beta)\left(w_{2}-1\right) x_{2}-\alpha x_{3}+(\alpha+\beta) w_{1} \\
\beta\left(1-w_{2}\right) x_{2}-\beta w_{1}
\end{array}\right] x_{1}
$$


Here $x_{1} \geq 0$ as it is a squared quantity. This is the form of the equations that we will work with. This set of equations appears very simple: such equations are called bilinear. The right hand side is linear in $x_{1}$ and also linear in combinations of $x_{2}$ and $x_{3}$, but is not linear in totality.

As an extension, a nonlinear plant can also be worked upon and a set of equations similar to (9) above can be developed. For a family of systems nonlinear in the state $y_{1}$ and affine in the control $u$ given by

$$
\dot{y}_{1}=y_{1} f\left(y_{1}^{2}\right)+b u
$$

a development similar to that undertaken above will result in a set of equations given by:

$$
\frac{d}{d t}\left[\begin{array}{l}
x_{1} \\
x_{2} \\
x_{3}
\end{array}\right]=\left[\begin{array}{c}
2\left(g\left(x_{1}\right)+b x_{2}\right) \\
(\alpha+\beta)\left(w_{2}-1\right) x_{2}-\alpha x_{3}+(\alpha+\beta) w_{1} \\
\beta\left(1-w_{2}\right) x_{2}-\beta w_{1}
\end{array}\right] x_{1}
$$

where $g\left(x_{1}\right)=f\left(y_{1}^{2}\right)$.

It can be seen that the linear case is the simplest form of this family of plants.

The set of equations (9) is in a bilinear form. The most notable system of this form is the Volterra-Lotka model [14] of interacting populations which is slightly different from (9) and is written in terms of the various populations $x_{j}(j=1,2,3 \ldots n)$ as:

$$
\dot{X}_{j}=\left(\sum_{i \neq j} a_{i} X_{i}\right) X_{j}
$$

This equation (12) is also bilinear but the rate of each population depends linearly on itself and not on one variable $x_{1}$ as in (9) or (11). This is a crucial difference and makes 
these equations categorically different from each other. The Volterra-Lotka model has been studied extensively and has been developed into a canonical format [15], and varied nonlinear analyses have been applied to it [16], [17], [18] in order to study features of practical issues like endemics, epidemics, vaccination projects, predator-prey models and other related cases. A variant of the Volterra-Lotka format was used by [19] to model the spread of a communicable disease in an isolated population which can be called a 2 variable analog to our system (9).

If $x_{1}$ represents the number of infected individuals and $x_{2}$ represents the number of uninfected or 'susceptible' individuals, then the rate at which the infected population changes is due to the interaction between the two groups $b x_{1} x_{2}$ and the number of people dying due to the disease $-a x_{1}$. Hence:

$$
\dot{x}_{1}=-a x_{1}+b x_{1} x_{2}
$$

Similarly the rate of change of the number of uninfected individuals is the interaction term $b x_{1} x_{2}$. Hence we have:

$$
\dot{x}_{1}=-b x_{1} x_{2}
$$

It can be seen that (13) possesses an equilibrium set given by $S=\left\{x \in \mathbb{R}^{2}: x_{1}=0\right\}$ which intuitively means that the spread of the disease is stable when there is no disease, irrespective of the size of the total population. 


\section{CHAPTER III}

\section{EQUILIBRIUM SETS, INVARIANT SETS AND STABILITY}

\section{A qualitative understanding of the system}

The nature of the solution of a system of differential equations can be understood from its nullclines and equilibrium points. We rewrite our system of equations (9) below for convenience after substituting $a, b, c \ldots g$ for the various coefficients in (9):

$$
\frac{d}{d t}\left[\begin{array}{l}
x_{1} \\
x_{2} \\
x_{3}
\end{array}\right]=\left[\begin{array}{c}
-a+b x_{2} \\
c x_{2}+d x_{3}+e \\
f x_{2}+g
\end{array}\right] x_{1} \quad \forall x_{1} \geq 0
$$

From (14) one can also derive an informal understanding of the dynamics of the set of equations. Firstly $x_{1}$ is the square of the plant output, hence as the output is regulated the rates of development of the gains becomes slow once the plant output is smaller than one. One can suitably scale the plant output to take advantage of this feature. In the above, we did not take into account the change due to the development of $x_{2}$, and $x_{3}$ as denoted by the quantities in the parentheses in (14). The parentheses contain the nullclines of the system. Any trajectory through a sample point $\left\{x_{1}, x_{2}, x_{3}\right\}$ in the 3 -space of $x_{i}$ 's shall move rapidly if it is away from these nullclines. As this sample point approaches one of the nullclines, the corresponding $x_{i}$ slows down and stops as it touches the nullcline, but as other rates are non-zero, the trajectory moves ahead. 
Another concern due to time-varying gains might be the occurrence of trivial feedback gain which would result in trivial control input even in the presence of non-zero measurement. However in (14) $x_{2}$ represents the feedback gain and the arrangement of the nullclines again assures that a trajectory shall not get trapped in the plane represented by $x_{2}=0$ and shall cross it. Hence the feedback gain will be zero momentarily which can be allowed for stable plants, but will be undesirable for unstable ones.

We find that the entire plane $x_{1}=0$ comprises of equilibrium points of the system (14). There cannot be other equilibrium points of the system without implying $x_{1}$ and $x_{3}$ are identical. The occurrence of a set of non-isolated equilibrium points is unusual for control engineering problems, which usually have a few isolated equilibrium points. The occurrence of the equilibrium set can be attributed to the dependence of each equation on $x_{1}$. This is the crucial difference that made our set of equations different from the Volterra-Lotka equations (12), which have a trivial equilibrium point at the origin and another (if possible) at the intersection of the nullclines $\sum_{i \neq j} a_{i} x_{i}=0$. Hence the equations appear similar in form but they are of remarkably different nature.

The occurrence of an equilibrium set warrants the extension of standard definitions of stability and the theorems that are used to prove stability of systems in the presence of isolated equilibrium points which are presented next. 


\section{Few definitions}

The occurrence of equilibrium sets in control engineering problems is unusual. However extensions of most concepts associated with isolated equilibrium points are available.

For the dynamical system represented by the following ordinary differential equation

$$
\dot{x}=f(x) \quad \forall x \in \mathbb{R}^{n}
$$

we present the following definitions [20]:

DEFINITION 1: The subset $\Gamma$ of an equilibrium set $S=\left\{x \in \mathbb{R}^{n}: f(x)=0\right\}$ of the system of equations (15) is called a connected branch of $S$, if for any $x_{1}, x_{2} \in S$ there exists a continuous curve in $\Gamma$ connecting $x_{1}$ and $x_{2}$, and if there exists a positive number $\delta$ such that there is no other equilibrium point of the system except $\Gamma$ itself in the open neighborhood $B(\delta, \Gamma)=\left\{x \in \mathbb{R}^{n}: d(x, \Gamma)<\delta\right\}$ of $\Gamma$, where $d(x, \Gamma)=\inf _{y \in \Gamma}\|x-y\|$ denotes the distance from $x$ to $\Gamma$.

DEFINITION 2: The connected branch of equilibrium set $\Gamma$ is stable if for any $x_{0}=x\left(t_{0}\right)$ and any scalar $\varepsilon>0$ there exists a $\delta>0$ such that if $d\left(x\left(t_{0}\right), \Gamma\right)<\delta$ then $d(x(t), \Gamma)<\varepsilon$ for $t>t_{0}$.

DEFINITION 3: The connected branch of equilibrium set $\Gamma$ is asymptotically stable if in addition to being stable it has the property that $d(x(t), \Gamma) \rightarrow 0$ as $t \rightarrow \infty$ if $d\left(x\left(t_{0}\right), \Gamma\right)<\delta$. 
Invariant sets are a more generic concept and they incorporate equilibrium sets as a special subset. We need the concept of invariant sets as stability results for non-isolated equilibria are defined in terms of invariant sets. We present the following definitions from [21]:

DEFINITION 4: A set $\mathcal{M}$ is invariant with respect to a dynamical system (15) if

$$
x(0) \in \mathcal{M} \Rightarrow x(t) \in \mathcal{M}, \forall t \in \mathbb{R}
$$

DEFINITION 5: A set $\mathcal{M}$ is positively invariant with respect to a dynamical system (15) if

$$
x(0) \in \mathcal{M} \Rightarrow x(t) \in \mathcal{M}, \quad \forall t>0 .
$$

Hence all equilibrium points and sets are covered in invariant sets. However, motion is allowed within the invariant sets (e.g. limit cycles and other closed paths) whereas equilibrium sets are defined by the states where the time derivative $\dot{x}=0$, and no motion is allowed. 


\section{Stability in the presence of equilibrium sets}

Lyapunov's methods are the standard method to prove stability of dynamical systems. However Lyapunov's methods are not applicable to our system of interest (14). Lyapunov's theorem (or Lyapunov's direct method) requires the candidate Lyapunov function to be positive definite in the region of interest, but the existence of non-isolated equilibrium points poses problems in this regard. Hence this method is not applicable to cases with non-isolated equilibrium points. Lyapunov's indirect method (if applicable) also fails for our problem as the matrix that results from linearization about any equilibrium point possesses zero eigenvalues and the indirect method fails to comment about such cases.

To prove stability for cases with equilibrium sets one uses LaSalle's invariance theorem which is stated below [21]:

THEOREM 1: Let $D \subset \mathbb{R}^{n}$ be a domain for the system of equations (15). Let $\Omega \subset D$ be a compact set that is positively invariant with respect to (15). Let $V: D \rightarrow \mathbb{R}$ be a continuously differentiable function such that $\dot{V}(x) \leq 0$ in $\Omega$. Let $E$ be the set of all points in $\Omega$ where $\dot{V}(x)=0$. Let $M$ be the largest invariant set in $E$. Then every solution of (15) starting in $\Omega$ approaches $M$ as time $t \rightarrow \infty$. 
Note that LaSalle's invariance principle holds for a more general case of the occurrence of invariant sets, which include connected branches of equilibrium sets and/or limit cycles.

LaSalle's theorem generalizes Lyapunov's theorem for invariant sets, but it also relaxes the need of the positive definiteness of the Lyapunov function $V(x)$ and the negative definiteness of $\dot{V}(x)=0$ for asymptotic stability as required in Lyapunov's Theorem. The compact set also provides a notion of the domain of attraction for the invariant set as well, which is not given by Lyapunov's theorem.

LaSalle's result does not provide any help in guessing the Lyapunov-like function $V(x)$. On the contrary the relaxations dilate the set of possible Lyapunov-like functions. In fact one is confronted with another issue of finding a compact set which is positively invariant to start with. Finding a positively invariant compact set involves discovering surfaces that the flow shall not cross. In this regard, one needs to fall back on the nature of the solution. But as one does not know the exact solution of most sets of differential equations one has to devise a workaround which is the subject of the next chapter. 


\section{CHAPTER IV}

\section{FIRST INTEGRALS OF DIFFERENTIAL EQUATIONS}

\section{What are first integrals?}

DEFINITION 1: For the ordinary differential equation system

$$
\dot{x}=f(x) \quad \forall x \in \mathbb{R}^{n}
$$

a continuously differentiable function defined over a domain $D \subset \mathbb{R}^{n}, I: D \rightarrow \mathbb{R}$ is a first

integral if $I(x)$ is non-constant over the field and the time derivative $\dot{I}(x)=0$. Hence a first integral can be considered a quantity which is conserved over the motion governed by (16) in the domain $D$. Note that time dependent first integrals also exist, and remain constant over the solution of the system.

Any constant is a trivial first integral. A system that has a first integral on the whole $\mathbb{R}^{n}$ is

called a conservative system. It is not necessary that all ordinary differential equations possess a first integral [22]. At the same time a system of equations might have multiple independent first integrals as well. If $I(x)$ is a first integral then $I(x)+c, c . I(x), \log (I(x))$ etc. are called dependent first integrals. $c \in \mathbb{R}$ is a constant.

A first integral is useful because it connects its level curves and trajectories of the system [23]. A trajectory will lie completely on a level surface of a time-independent first integral. Hence each level curve of the first integral is a union of trajectories. Uniqueness 
of the solution assures that the union is disjoint. In fact, for systems in $\mathbb{R}^{2}$ any trajectory is a first integral. Hence the entire phase portrait can be identified from the level surfaces of the first integral.

\section{Need of first integrals}

Our interest in first integrals is due to two reasons. Firstly, the property that they remain constant over time can help us in finding Lyapunov-like functions. The use of first integrals to prove stability through Lyapunov's theorem and its corollaries is wellestablished [24]. Note that using a first integral for a Lyapunov-like function $V(x)$ means that we are looking for a stricter condition where the time derivative $\dot{V}(x)=0$ always than what is necessary for LaSalle's theorem $\dot{V}(x) \leq 0$. This might make the task of identifying suitable Lyapunov-like functions all the more difficult.

However extensions to cases which require LaSalle's theorem call for and utilize a second property: that a trajectory cannot cross a level curve of the first integral. This provides us with a method to identify surfaces which can be used for constructing positively invariant sets by using a higher level curve as one of the boundaries of the set in question.

Finding Lyapunov and Lyapunov-like functions has always been an intriguing task and till date intuition and experience are the surest tools for this purpose. But we encountered 
earlier that a system of equations can have multiple first integral. Hence if we have a method to find first integrals, then this tradeoff for a stricter situation can help us.

\section{A method to find first integrals}

A general method to find first integrals for any kind of ordinary differential equation does not exist. However a method for equations in $\mathbb{R}^{3}$ exists. Note that this does not imply that all differential equations in $\mathbb{R}^{3}$ will have at least one first integral. This method can find a first integral only if it exists.

The method to find first integrals for a system of ordinary differential equations is called the compatibility analysis and is based on Fröbenius Integrability Theorem [22]. It allows a method to find conditions on the parameters of a vector field for the existence of timeindependent first integrals and provides an explicit method for their computation. The calculations involved are quite involved and the computation nearly always requires a computer algebra system. For our calculations we have used the commonly available technical software Maple. We introduce the method in the following pages, but first we discuss some preliminaries [22] on the next page: 
DEFINITION 2: For a system of differential equations (16) a differentiable vector field is defined as:

$$
\delta_{\mathrm{f}}=\mathrm{f} . \partial_{\mathrm{x}}=\left[\begin{array}{c}
\mathrm{f}_{1} \\
\vdots \\
\mathrm{f}_{n}
\end{array}\right] \cdot\left[\begin{array}{c}
\frac{\partial}{\partial x_{1}} \\
\vdots \\
\frac{\partial}{\partial x_{n}}
\end{array}\right]
$$

Note this definition is analogous to the definition of a Lie Derivative.

DEFINITION 3: The Lie Bracket between two vector fields is defined as:

$$
[\mathrm{f}, \mathrm{g}]=\delta_{\mathrm{f}} \mathrm{g}-\delta_{\mathrm{g}} \mathrm{f}
$$

DEFINITION 4: Two vector fields $\delta_{\mathrm{f}}, \delta_{\mathrm{g}}$ in $\mathbb{R}^{3}$ are called compatible if:

$$
\operatorname{det}\{\mathrm{f}, \mathrm{g},[\mathrm{f}, \mathrm{g}]\}=0
$$

THEOREM 1 (Fröbenius) [22]: Assume that the two vector fields $\delta_{\mathrm{f}}$ and $\delta_{\mathrm{g}}$ in $\mathbb{R}^{3}$ admit the same non-trivial globally defined time-independent first integral $I(x)$, that is,

$$
\text { f. } \partial_{\mathrm{x}} I=\mathrm{g} . \partial_{\mathrm{x}} I=0
$$

Then the three vector field $\mathrm{f}, \mathrm{g}$ and $[\mathrm{f}, \mathrm{g}]$ are linearly dependent for all points $x \in \mathbb{R}^{3}$, which implies:

$$
\operatorname{det}\{\mathrm{f}, \mathrm{g},[\mathrm{f}, \mathrm{g}]\}=0
$$


The compatibility condition implies that the two vector fields are considered parallel (at least locally). Hence a local first integral to one system is admissible to the other as well.

The algorithm to find first integrals comprises of the following steps:

1) Find a linear vector field $\delta_{\mathrm{g}}$ compatible with $\delta_{\mathrm{f}}$

2) Find two first integrals $u(x), v(x)$ of $\delta_{g}$.

3) Compute $G(u, v)=\frac{\left(\mathrm{f} . \partial_{x} u\right)}{\left(\mathrm{f} . \partial_{x} v\right)}$

4) Find a first integral $I=I(u, v)$ of the first order equation $\frac{d u}{d v}=G(u, v)$

Essentially for a given $\delta_{\mathrm{f}}$, this method finds a vector field $\delta_{\mathrm{g}}$ with known first integrals for which the compatibility condition with $\delta_{\mathrm{f}}$ is satisfied. Then it finds out first integrals for the vector field $\delta_{\mathrm{g}}$ and using Frobenius' theorem it tries to find out a common integral which is applicable to the original system. Since the vector field $\delta_{\mathrm{g}}$ is a linear vector field in $\mathbb{R}^{3}$, it is relatively easier to find out first integrals for $\delta_{\mathrm{g}}$.

One of the important points in this method is the fact that $G(u, v)$ is a function of $u, v$ only, which follows from the compatibility condition and the fact that $G(u, v)$ itself is a first integral of $\mathrm{f}$. The result of the last integration: $I(u, v)=I(u(x), v(x))$ is a first integral which is common to both vector fields, and is the first integral that we have been looking for. 
The compatibility condition method is advantageous over other methods in the fact that it is constructive: it does not reject candidate functions, but it yields a first integral if the system of equations permits one. Also this method can find transcendental first integrals as well, unlike other methods.

\section{Finding a first integral to our problem}

Our aim is to find a first integral (if it exists) for the following system of ordinary differential equations

$$
\frac{d}{d t}\left[\begin{array}{l}
x_{1} \\
x_{2} \\
x_{3}
\end{array}\right]=\left[\begin{array}{c}
-a+b x_{2} \\
c x_{2}+d x_{3}+e \\
f x_{2}+g
\end{array}\right] x_{1} \quad \forall x_{1} \geq 0
$$

Assume that there exists a linear vector field $\delta_{\mathrm{g}}$ corresponding to the system of equations in $\mathbb{R}^{3}$ :

$$
\dot{x}=g(x)
$$

Where $g(x)$ depends linearly on $x$ through a $3 \times 3$ matrix L with constant coefficients

$$
g(x)=\mathrm{L} x
$$

The compatibility condition between $\delta_{\mathrm{f}}$ and $\delta_{\mathrm{g}}$ attains the form of:

$$
\operatorname{det}\left[\begin{array}{ccc}
x_{1}\left(-a+b x_{2}\right) & L_{11} x_{1}+L_{12} x_{2}+L_{13} x_{3} & P\left(x_{1}, x_{2}, x_{3}\right) \\
x_{1}\left(c x_{2}+d x_{3}+e\right) & L_{21} x_{1}+L_{22} x_{2}+L_{23} x_{3} & Q\left(x_{1}, x_{2}, x_{3}\right) \\
x_{1}\left(g+f x_{2}\right) & L_{31} x_{1}+L_{32} x_{2}+L_{33} x_{3} & R\left(x_{1}, x_{2}, x_{3}\right)
\end{array}\right]=0, \quad \forall \mathrm{x} \in \mathbb{R}^{3}
$$

In the above expression, $P, Q, R$ are quadratic homogenous polynomials defined by:

$$
[\mathrm{f}, \mathrm{g}]=(P, Q, R)
$$


The compatibility condition (23) is a polynomial of fifth degree in $\mathrm{x}$ and of second degree in the unknowns $L_{i j}(i, j=1,2,3)$. On collecting the coefficients of terms with same power of $\mathrm{x}$ and equating them to zero we get a set of eighteen nontrivial simultaneous equations quadratic in $L_{i j}$ which are given on the following pages:

$$
\begin{aligned}
& \operatorname{af} L_{21}{ }^{2}+e f L_{11} L_{21}-d g L_{11} L_{31}-a d L_{31}{ }^{2}-b e L_{21} L_{31}-c g L_{11} L_{21}-a c L_{21} L_{31}+ \\
& \text { b } g L_{21}{ }^{2}=0
\end{aligned}
$$

$b d L_{31}^{2}-d f L_{11} L_{31}=0$

$d f L_{11} L_{21}-b d L_{21} L_{31}=0$

$f^{2} L_{11} L_{23}-f^{2} L_{13} L_{21}+c^{2} L_{12} L_{31}-b c L_{22} L_{31}-c f L_{12} L_{21}-c^{2} L_{11} L_{32}-c f L_{11} L_{33}$

- bf $L_{23} L_{31}+c f L_{13} L_{31}-d f L_{12} L_{31}+c f L_{11} L_{22}+b c L_{21} L_{32}+b f L_{21} L_{33}+2 b d L_{31} L_{32}$

$-d f L_{11} L_{32}=0$

$d^{2} L_{12} L_{31}+d f L_{13} L_{21}-b d L_{21} L_{33}-b d L_{23} L_{31}-d^{2} L_{11} L_{32}+d f L_{11} L_{23}=0$

$2 b d L_{31} L_{33}-2 b d L_{22} L_{31}+2 d f L_{11} L_{22}-2 d f L_{11} L_{33}-2 c d L_{11} L_{32}+2 c d L_{12} L_{31}=0$

b g $L_{21} L_{33}-2$ b e $L_{22} L_{31}-b g L_{23} L_{31}-2 a c L_{21} L_{32}+2 a f L_{21} L_{22}-a f L_{21} L_{33}+a f L_{23}$ $L_{31^{-}} 2$ ad $L_{31} L_{32}-2$ ce $L_{11} L_{32}-c g L_{11} L_{33}+2 c e L_{12} L_{31}+c g L_{13} L_{31}-d g L_{11} L_{32^{-}} 2 c$ 
$g L_{12} L_{21}-d g L_{12} L_{31}+2 b g L_{21} L_{22}+2$ ef $L_{11} L_{22}-e f L_{11} L_{33}+e f L_{13} L_{31}+2 f g L_{11} L_{23}$ $-2 f g L_{13} L_{21}=0$

$-2 d g L_{11} L_{33}-b$ e $L_{23} L_{31}-a d L_{21} L_{32}+2 a f L_{21} L_{23}+a d L_{22} L_{31}-a c L_{21} L_{33}-a c L_{23}$ $L_{31-} 2 a d L_{31} L_{33}-2 d$ e $L_{11} L_{32}-b$ e $L_{21} L_{33}-c g L_{13} L_{21}-d g L_{12} L_{21}+d g L_{11} L_{22}-c g$ $L_{11} L_{23}+2 b g L_{21} L_{23}+2 d e L_{12} L_{31}+e f L_{11} L_{23}+e f L_{13} L_{21}=0$

$-e^{2} L_{11} L_{32}+e^{2} L_{12} L_{31}+g^{2} L_{11} L_{23}-g^{2} L_{13} L_{21}-a e L_{21} L_{32}-a g L_{21} L_{33}+a e L_{22} L_{31}+a g$ $L_{23} L_{31}+e g L_{11} L_{22}-\operatorname{eg} L_{12} L_{21}-\operatorname{eg} L_{11} L_{33}+e g L_{13} L_{31}=0$

$-f^{2} L_{13} L_{22}-c f L_{12} L_{33}+b c L_{11} L_{32}+b d L_{32}^{2}+f^{2} L_{12} L_{23}-d f L_{12} L_{32}-b c L_{12} L_{31}-b^{2}$ $L_{21} L_{32}+b f L_{22} L_{33}+b f L_{21} L_{12}+b^{2} L_{22} L_{31}+c f L_{13} L_{32}-b f L_{22} L_{11}-b f L_{23} L_{32}=0$

$d f L_{13} L_{23}-b d L_{23} L_{33}-d^{2} L_{13} L_{32}+d^{2} L_{12} L_{33}=0$

$-b^{2} L_{21} L_{33}-c^{2} L_{13} L_{32}+c^{2} L_{12} L_{33}+2 b d L_{32} L_{33}+b^{2} L_{23} L_{31}+b f L_{21} L_{13}-b f L_{23} L_{11}+$ $b c L_{23} L_{32}-b c L_{13} L_{31}+b d L_{11} L_{32}-b d L_{12} L_{31}+b c L_{11} L_{33}+d f L_{12} L_{22}-2 d f L_{12} L_{33}$ - $f L_{12} L_{23}+c f L_{13} L_{22}-b c L_{22} L_{33}-b d L_{22} L_{32}=0$

$a f L_{22}^{2}-a d L_{32}^{2}+b g L_{22}^{2}+b g L_{21} L_{12}-2 a b L_{22} L_{31}-b g L_{22} L_{11}+b g L_{22} L_{33}$ $+2 a b L_{21} L_{32}-b g L_{23} L_{32}+b$ e $L_{11} L_{32}-b e L_{12} L_{31}+a f L_{22} L_{11}-a c L_{22} L_{32}-a f L_{22} L_{33}$ $a L_{21} f L_{12}+a L_{23} f L_{32}-a c L_{11} L_{32}+a c L_{12} L_{31}-c L_{12} L_{33} g+c L_{13} g L_{32}+2 f L_{12} L_{23} g-$ $2 f g L_{13} L_{22}-d g L_{12} L_{32}-c g L_{12} L_{22}+e f L_{12} L_{22}-e f L_{12} L_{33}-b$ e $L_{22} L_{32}+e f L_{13} L_{32}=0$ 
$b d L_{33}^{2}+2 d f L_{13} L_{22}-2 b d L_{22} L_{33}-b d L_{13} L_{31}-d f L_{13} L_{33}+b d L_{11} L_{33}+2 c d L_{12} L_{33}-$ $2 c d L_{13} L_{32}=0$

$-a d L_{11} L_{33}+b g L_{23}^{2}+a d L_{13} L_{31}+a d L_{22} L_{33}-a d L_{33}^{2}+e f L_{13} L_{23}+a f L_{23}{ }^{2}$

- $a d L_{23} L_{32}-2 d e L_{13} L_{32}+d g L_{13} L_{22}-b e L_{23} L_{33}-c g L_{13} L_{23}-d g L_{13} L_{33}-d g L_{12} L_{23}$

- ac $L_{23} L_{33}+2$ de $L_{12} L_{33}=0$

$-2 a b L_{23} L_{31}+a c L_{13} L_{31}+b g L_{21} L_{13}-b g L_{23} L_{11}+b e L_{11} L_{33}-b e L_{13} L_{31}+2 a b L_{21}$ $L_{33}+2$ ef $L_{13} L_{22}+2$ af $L_{22} L_{23}-a f L_{21} L_{13}-2 a d L_{32} L_{33}+a f L_{23} L_{11}-2 a c L_{23} L_{32}-a c$ $L_{11} L_{33}-2 \operatorname{cg} L_{12} L_{23}-a d L_{11} L_{32}-2 b$ e $L_{22} L_{33}+a d L_{12} L_{31}-2 c e L_{13} L_{32}$

$+2 b g L_{22} L_{23}-2 d g L_{12} L_{33}+2 c e L_{12} L_{33}=0$

$a^{2} L_{22} L_{31}-a^{2} L_{21} L_{32}+g^{2} L_{12} L_{23}-g^{2} L_{13} L_{22}+a$ e $L_{12} L_{31}+\operatorname{ag} L_{22} L_{11}-a g L_{21} L_{12}$ $+\operatorname{ag} L_{23} L_{32}-\operatorname{ag} L_{22} L_{33}-\operatorname{ae} L_{11} L_{32}-\operatorname{eg} L_{12} L_{33}+\operatorname{eg} L_{13} L_{32}=0$

$a^{2} L_{23} L_{31}-a^{2} L_{21} L_{33}-e^{2} L_{13} L_{32}-a$ e $L_{23} L_{32}+$ a e $L_{22} L_{33}-a g L_{21} L_{13}+$ a e $L_{13} L_{31}$ $+e^{2} L_{12} L_{33}-\operatorname{eg} L_{12} L_{23}+\operatorname{ag} L_{23} L_{11}-a e L_{11} L_{33}+\operatorname{eg} L_{13} L_{22}=0$

(The complete set of eighteen equations shall be referred to as equation (25).)

The above equations were solved using the Maple computer algebra system to yield several nontrivial solutions, of which the following was chosen due to its symmetry for further calculations: 


$$
\mathrm{L}=\left[\begin{array}{ccc}
0 & \frac{b}{f} \lambda & 0 \\
0 & K \lambda & 0 \\
0 & \lambda & 0
\end{array}\right] \text { where } f K^{2}-c K-d=0
$$

Hence the compatible linear vector field is given by:

$$
\dot{x}=\mathrm{L} x
$$

where $\mathrm{L}$ is given by (26)

For the above system, the following were identified as first integrals:

$$
\begin{aligned}
& u(\mathrm{x})=b x_{3}-f x_{1} \\
& v(\mathrm{x})=b x_{3}-K f x_{1}
\end{aligned}
$$

Processing further we have the following first order equation in $u$ and $v$.

$$
\frac{d v}{d u}+\frac{b d}{K(b g+a f)} v=\left(\frac{b d}{b g+a f}\right) u+\left(\frac{b e+a f K}{b g+a f}\right)
$$

The solution (trajectory) of the above first order equation in $u$ and $v$ shall be a first integral as well:

$$
I(\mathrm{x})=\left(x_{2}-K x_{3}+\frac{K}{d}(g K-e)\right) \cdot \exp \left(A\left(x_{3}-\frac{f}{b} x_{1}\right)\right)
$$

where $(K f-c) K=d$ and $A=\frac{b d}{K(b g+a f)}$ 
The above is a first integral to our system of differential equations (21). There might exist other first integrals arising from the other nontrivial solutions of (25) as well.

Now in the next chapter we shall present a method which shall utilize a first integral for proving stability for systems of ordinary differential equations which possess a connected equilibrium set. 


\section{CHAPTER V \\ PROOF OF STABILITY}

\section{Outline}

Our system of differential equations (14) possesses a connected branch of equilibrium set on the $x_{1}=0$ plane. Hence this necessitates the use of LaSalle's theorem. For using LaSalle's theorem we need a method to develop positively invariant sets in the region of interest. This requires a method to identify surfaces which the flow of the equations will not cross in positive time. For this we rely on the understanding of the nature of the flow that can be derived from the form of the equations without attempting to solve them and on the first integrals that were developed using Frobenius's compatibility result.

Once the positively invariant sets are identified, the problem is not solved: as there are nullcline planes in the region of interest where the nature of the flow and hence the nature of the surfaces shall change, resulting in the fact that the positive invariance of the set might be lost. Hence there might be trajectories where the existence of such a positively invariant set cannot be shown. For such cases, we again rely on the nature of the flow and use a union of such sets to develop a superset which would contain the trajectory from its starting point to all future time, hence ensuring positive invariance. Once positive invariance is ensured, LaSalle's theorem ensures that the trajectories converge onto the invariant set which comprises of a section of the equilibrium set contained in the positively invariant set developed above. 


\section{A 2-dimensional example}

Before we discuss the full fledged 3-dimensional case represented by the set of equations given by (14), we shall first consider a 2-dimensional problem to understand the basic steps involved. For this purpose we consider a problem analogous to the spread of an infectious disease in a population, given by (13) in chapter II. Such a 2-dimensional problem can be derived from (14) by putting $x_{3} \equiv 0$ in it and rewriting it as:

$$
\begin{aligned}
& \dot{y}=a(x-c) y \quad \forall y \geq 0 \\
& \dot{x}=b x y
\end{aligned}
$$

Consider that $a, b, c$ are all positive. One can easily see that the above set of equations has the $\mathrm{x}$-axis as its equilibrium set. One can also divide (31a) by (31b) and solve for the trajectory passing through any 2 generic points $\left(x_{i}, y_{i}\right)$ and $\left(x_{f}, y_{f}\right)$ :

$$
\frac{y_{f}}{a}-\frac{x_{f}}{b}+\frac{c}{b} \ln \left|x_{f}\right|=\frac{y_{i}}{a}-\frac{x_{i}}{b}+\frac{c}{b} \ln \left|x_{i}\right|
$$

One can observe from various such trajectories that $S=\{(x, y): x \leq c, y=0\}$ is the stable set of equilibrium and $U=\{(x, y): x \geq c, y=0\}$ is the unstable set.

We can also show this analytically using LaSalle's Theorem:

Consider the domain $x \leq 0$, where we choose the following Lyapunov-like function:

$$
V(x, y)=\frac{y}{a}-\frac{x}{b}
$$


As $y$ is positive, the time derivative of this Lyapunov-like function is negative throughout:

$$
\dot{V}(x, y)=-c y \leq 0
$$

Hence if we choose the following compact set (see figure 5)

$$
\Omega=\left\{(x, y): x \leq 0, y \geq 0, V(x, y)=\left(\frac{y}{a}-\frac{x}{b}\right) \leq K, K>0 \text { is a constant }\right\}
$$

then it is positively invariant, as $V(x, y)$ is radially unbounded. (See [21] for explanation)

Now we find the set $E$ where the time derivative of $V(x, y)$ is zero is given as:

$$
E=\{(x, y):(x, y) \in \Omega, y=0\}
$$

We can also see that $E$ is an invariant set as well, hence we have:

$$
M=E=\{(x, y):(x, y) \in \Omega, y=0\}
$$

Therefore due to LaSalle's theorem, any trajectory starting in the set $\Omega$ shall approach $M$ as time tends to infinite.

Now consider the region with positive $x$. To construct a positively invariant set, we choose a starting point $\left(x_{o}, y_{o}\right)$ where $x_{o} \leq c$ as any trajectory starting with $x_{o}>c$ will not be bounded. Now we choose $y_{o}$ such that the trajectory passing through $\left(x_{o}, y_{o}\right)$ also passes through $(0, c)$ which implies:

$$
\frac{y_{o}}{a}-\frac{x_{o}}{b}+\frac{c}{b} \ln x_{o}=-\frac{c}{b}+\frac{c}{b} \ln c
$$




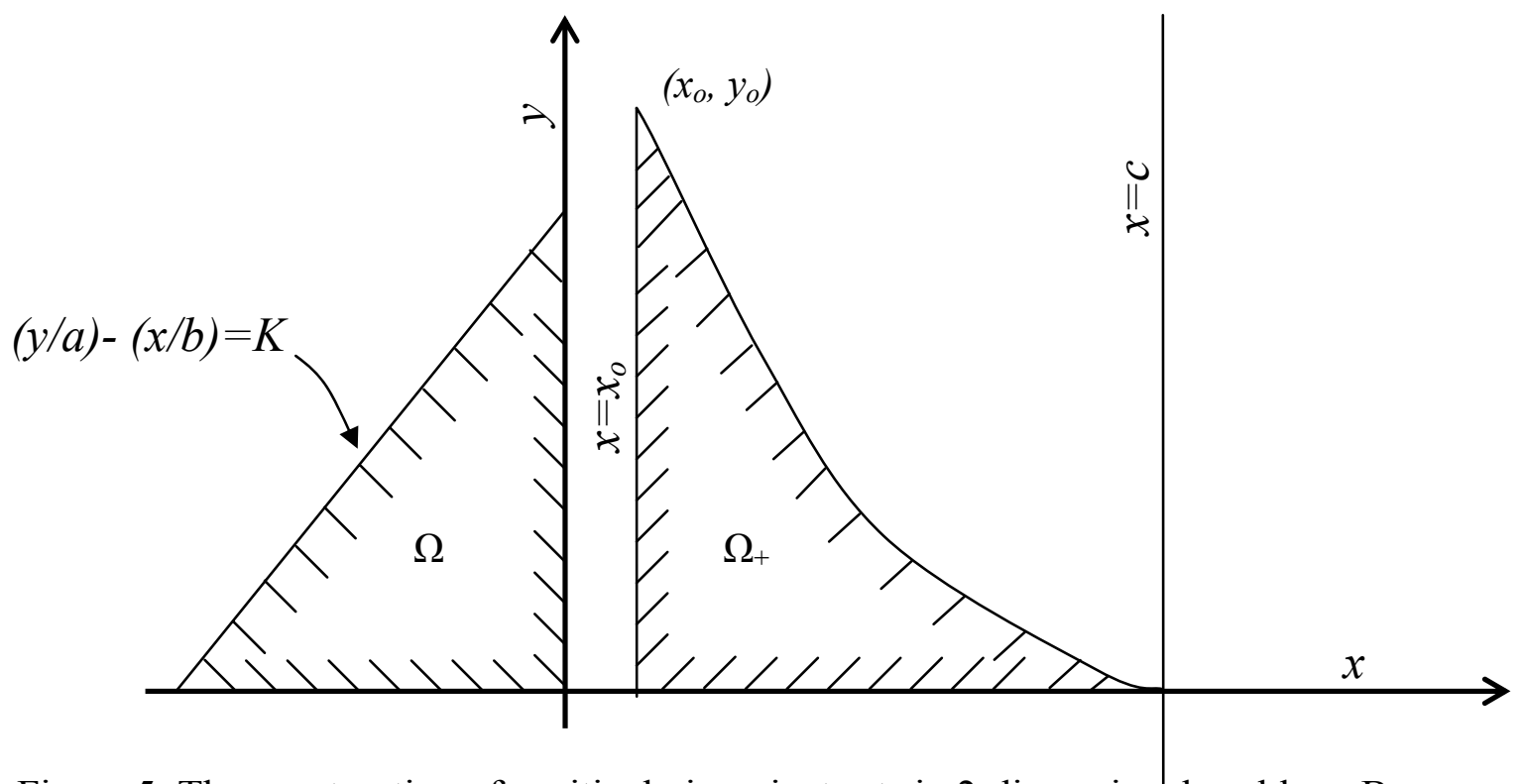

Figure 5: The construction of positively invariant sets in 2-dimensional problem. By increasing $K$ and by choosing $x_{o}$ sufficiently closer to the $x$ axis, one can cover the entire region of attraction.

Now consider the region $\Omega_{+}$in figure 5 bounded by (38a) and

$$
\begin{aligned}
& x_{o} \leq x \leq c \\
& y \geq 0
\end{aligned}
$$

This set $\Omega_{+}$is a compact and positively invariant set. We now choose the following Lyapunov-like function over $\Omega_{+}$whose time derivative is zero throughout $\Omega_{+}$:

$$
V(x, y)=\frac{y}{a}-\frac{x}{b}+\frac{c}{b} \ln x
$$

Hence in this case, we have $E=\Omega_{+}$. And the largest invariant set in $E$ is given as:

$$
M=\left\{(x, y):(x, y) \in \Omega_{+}, y=0\right\}
$$

Hence, by LaSalle's theorem, any trajectory starting within $\Omega_{+}$shall eventually approach the largest invariant subset $M$. By choosing a sufficiently large $K$ and $x_{o}$ arbitrarily close to the $\mathrm{y}$-axis one can show that that the set $S=\{(x, y): x \leq c, y=0\}$ is stable. 


\section{Construction of positively invariant sets for the complete problem}

Now we consider the complete 3-dimensional problem given by (14). We shall now discuss how to build a positively invariant set for (14) which is reproduced below:

$$
\frac{d}{d t}\left[\begin{array}{c}
x_{1} \\
x_{2} \\
x_{3}
\end{array}\right]=\left[\begin{array}{c}
-a+b x_{2} \\
c x_{2}+d x_{3}+e \\
f x_{2}+g
\end{array}\right] x_{1} \quad \forall x_{1} \geq 0
$$

On observing (39), one notes that, as $x_{l}$ is a positive quantity throughout, the sign of the right-hand-side of the equations and hence the flow is identified by the planes:

$$
\begin{array}{r}
b x_{2}-a=0 \\
c x_{2}+d x_{3}+e=0 \\
f x_{2}+g=0
\end{array}
$$

The planes represented by (42a), (42b), (42c) and $x_{I}=0$ are the nullclines for system (41). Henceforth, we shall refer to (42a), (42b) and (42c) as the nullcline planes, though the complete nullcline comprises of these planes and the $x_{1}=0$ plane. Interestingly, these nullcline planes are independent of $x_{1}$ and depend on only the states that have been introduced by the feedback loop. Hence we need to look at only the $x_{2^{-}} x_{3}$ planes (i.e. $x_{1}=$ any positive constant surface) to identify the flow of the 3 variables. Our re-declaring variables while writing (9) has ensured that the planes (42a) and (42b) are parallel to one

of the axes. Looking at the original equations (9), it is clear that (42b) and (42c) intersect on the $x_{2}$ axis. The exact positioning of the point depends on the user-defined values of 


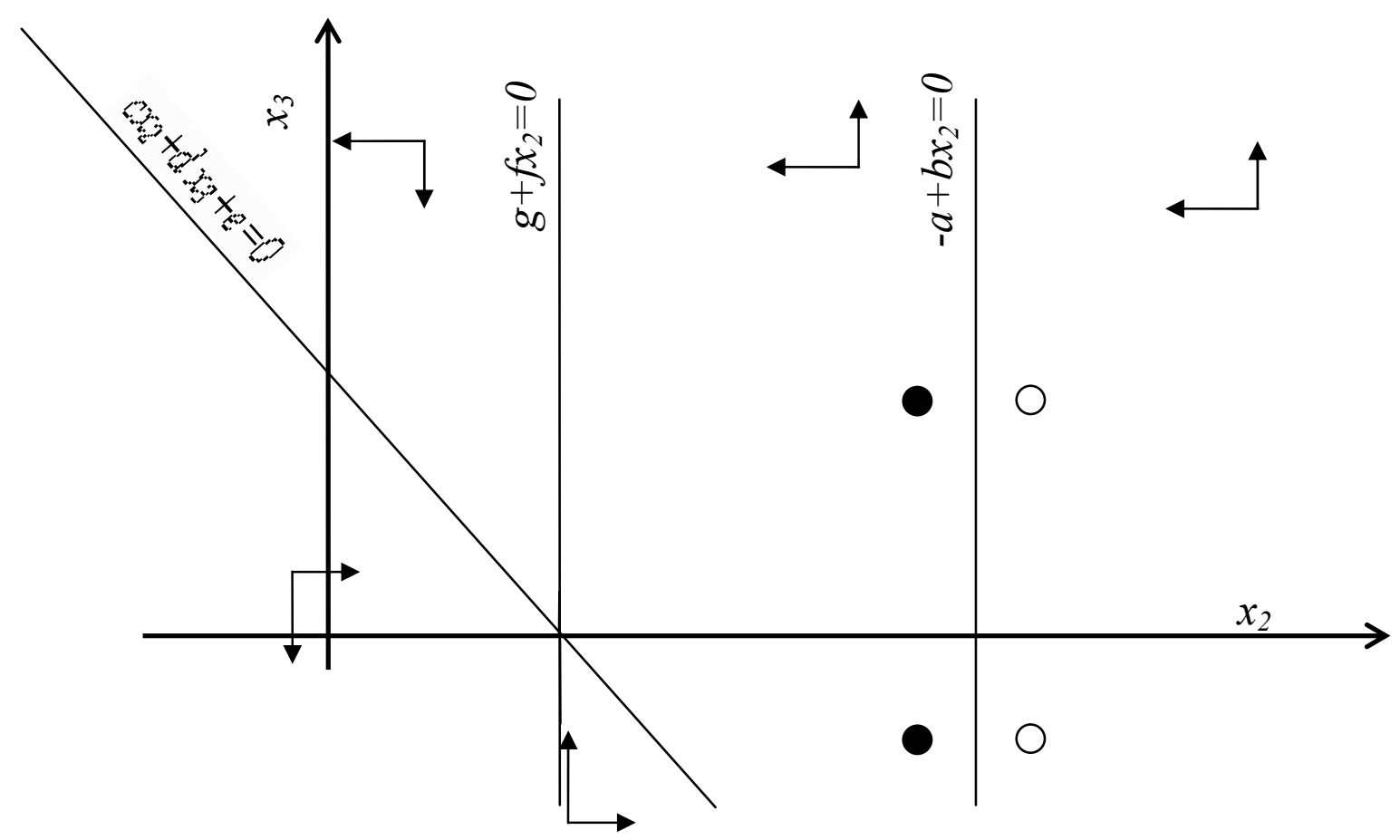

Figure 6: The nature of flow represented in any plane $x_{1}=$ positive constant The blank circle shows increasing $x_{1}$, while the solid circle shows decreasing $x_{1}$. The horizontal arrows show the direction of $x_{2}$ and the vertical arrows are for $x_{3}$.

$w_{1}$ and $w_{2}$ in (5). We can always choose the parameters to ensure that planes represented by (42b) and (42c) intersect on that side of (42a) where $\dot{x}_{1}=\left(b x_{2}-a\right) x_{1}<0$.

Figure 6 shows the three planes, as straight lines as they would appear on any $x_{2}-x_{3}$ plane. Equation (42a) defines the boundary between the regions where $x_{1}$ increases or decreases: the blank circle in the figure above represents motion of $x_{1}$ coming out of the plane of the figure, i.e. increasing, while the solid circle represents a decrease in $x_{1}$ and hence motion into the plane of the figure. The horizontal arrows in Figure 5 represent the direction in which $x_{2}$ would move, and similarly the vertical arrows represent the direction for $x_{3}$. 


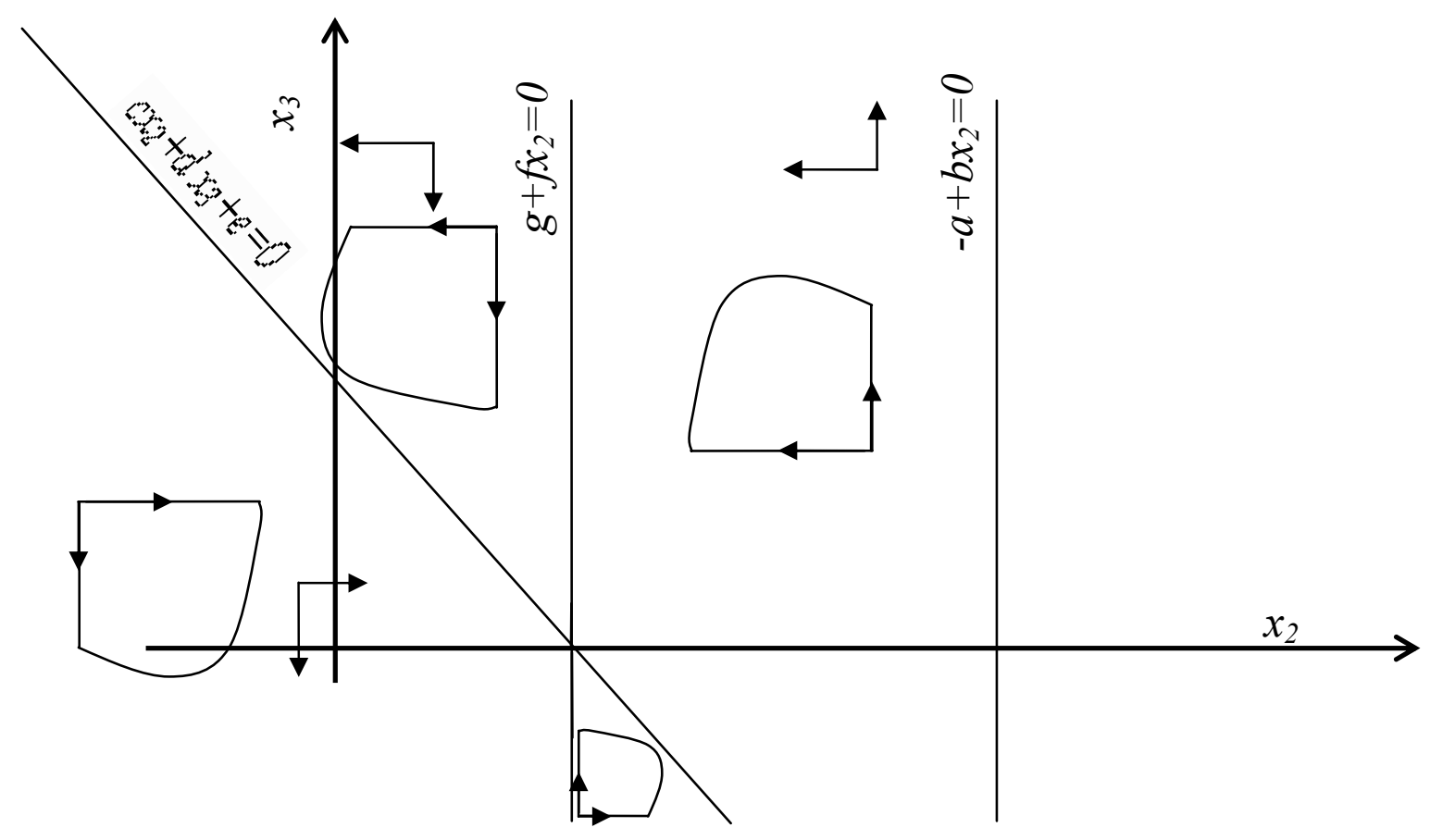

Figure 7: Construction of compact positively invariant sets in the various regions demarcated by the nullcline planes in the 3-dimensional problem. Only the "footprint" on the $x_{1}=0$ plane is shown above.

The direction of motion of $x_{2}$ is determined by the side of the plane represented by (42b) on which the sample point lies. Motion along $x_{2}$ shall be zero on the plane represented by (42b). Similarly as a sample point crosses the plane represented by (42c) the direction of motion of $x_{3}$ is also reversed. Note that a different choice of parameters might result in other configurations of the arrows. However the analysis will be on similar lines as presented here.

Now if we imagine planes perpendicular to the figure but along the arrows of the crosshair, then any trajectory starting from a point between the arrows will never intersect these planes, as the direction of the flow of the system are away from these planes. Now 
consider a trajectory starting from $x(0)=\bar{x}$ where $\bar{x}$ lies to the left of the plane given by (42a) in Figure 6. Consider the following set in non-negative $x_{1}$ half space of $\mathbb{R}^{3}$

$$
\Omega_{\bar{x}}=\left\{\begin{array}{l}
\text { region including and bounded by the planes } \\
x_{1}=0, x_{2}=\bar{x}_{2}, x_{3}=\bar{x}_{3} \text { and } I(x)=I(\bar{x})
\end{array}\right\}
$$

As $x_{1}$ is decreasing on this side of the plane represented by (42a), and the first integral $I(x)$ is continuous, it assures that $I(x)$ and each of the three planar boundaries in (43) have a continuous intersection. This ensures that each boundary is closed and the set is also closed.

It is difficult to show the above 3-dimensional sets on paper, but we represent their footprint on the $x_{1}=0$ plane as shown in Figure 7 above: the lines along the cross hair pictographically represent the planar boundaries and the smooth curve represents the first integral. Hence the set $\Omega_{\bar{x}}$ has three planes intersecting at the vertex $\left(\bar{x}_{1}, \bar{x}_{2}, 0\right)$ and is closed by the level curve of the first integral $I(x)$ passing through $\bar{x}$.

It can be seen that if $\Omega_{\bar{x}}$ does not intersect any of the nullcline planes then it is a positively invariant set as the level curves of the first integral are disjoint, and the flow moves away from the surfaces of the planes along $x_{2}$ and $x_{3}$ directions. Also such positively invariant sets are compact, as discussed above. Hence we develop positively invariant and compact sets in the flow of (41) Cases where such a set cannot be developed are discussed later. 


\section{Main proof}

We shall prove that $\left\{x \in \mathbb{R}^{3}: x_{1}=0, b x_{2}-a \leq 0\right\}$ is the stable equilibrium set. We break up the proof into cases depending on the intersection of the trajectory with the nullcline planes, as the nature of planar boundaries changes over the nullcline planes. Following is the outline of proof:

Case 1: Trajectory does not intersect nullcline planes: Find a set $\Omega_{\bar{x}}$ in non-negative $x_{1}$ half space of $\mathbb{R}^{3}$ as outlined in the previous section.

Case 1a: Set does not intersect nullcline planes: If the set $\Omega_{\bar{x}}$ does not intersect the three planes given by (42a)-(42c) then it is a positively invariant compact set. And due to LaSalle's theorem, all trajectories starting within this set shall asymptotically approach the largest invariant subset, which is $\left\{x \mid x \subset \Omega_{\bar{x}}, x_{1}=0\right\}$.

Case 1b: Set intersects nullcline planes: If a set $\Omega_{\bar{x}}$ intersects the nullcline planes (42b) or $(42 \mathrm{c})$ then the intersecting boundary might allow the flow to cross them, as across the nullcline planes the direction of change of $x_{2}$ or $x_{3}$ is reversed. If a set $\Omega_{\bar{x}}$ cannot be constructed which does not intersect the nullcline planes, then a union of such sets will be necessary to provide a positively invariant compact set. We shall utilize a union of sets constructed on the lines of $\Omega_{\bar{x}}$ to get a positively invariant compact set: the boundaries would still be aligned along the flow arrows and the first integral. The part 


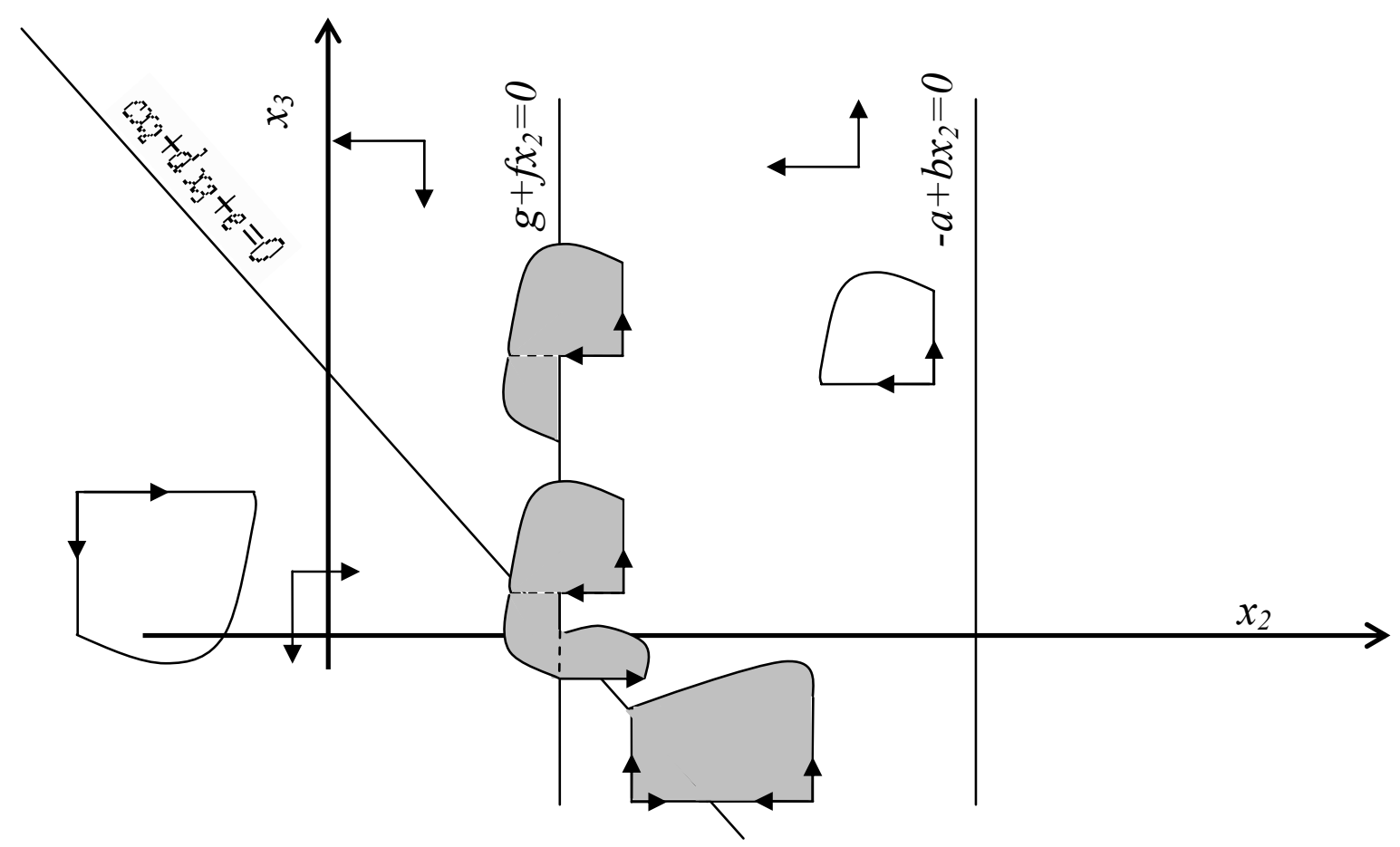

Figure 8: Construction of compact positively invariant sets by union. The leaking boundaries shown by dashed lines have been brought inside by union with other similarly constructed sets.

of the original boundary of $\Omega_{\bar{x}}$ which now has the flow going across it shall be brought into the interior of the union. To do this, we shall place another set which would be bounded by the same level curve of the first integral and planar boundaries along the direction of flow on this side of the nullcline plane as shown in the figure above. It can be shown that to develop a positively invariant set we will need a finite number of such unions. This can also be seen in figure 8 above. This is brought about if we choose the last set to have the point of intersection of (42b), (42c) and $x_{1}=0$ as one of its vertices, then the nature of the flow assures that these boundaries do not allow flow to cross them. Hence its compactness is not at stake. 
Case 2. Trajectory intersects nullcline planes: If the trajectory itself intersects the nullcline planes, then we can divide the trajectory into two parts: the trajectory from the starting point to the last intersection with the nullcline planes and the tail of the trajectory which is the part of trajectory from the last intersection onwards. The tail of the trajectory can be analyzed by Case 1 listed above. The trajectory from the initial point to the last intersection shall lie on a continuous level curve of the first integral and will be suitably bounded.

The possibility of the flow getting trapped in a closed path is unlikely as qualitatively there is little symmetry in the flow to make this possible. Extensions to Bendixson's negative criterion to systems of higher dimensions possessing first integrals [25] were applied but the negative criterion was not satisfied and hence a conclusion was not reached. A suitable analytical method is not available for identifying limit cycles and closed path in higher dimensions. As mentioned earlier, by observing the nature of the flow, the author considers it unlikely that there might be other closed orbits that might be a part of the invariant set. 


\section{CHAPTER VI}

\section{CONCLUSION}

This thesis discussed a control algorithm developed from the emotional processing network. From a control engineering outlook, we achieved a feedback loop in which the feedback gains were updated depending on the error. This control loop generates an equilibrium set. Intuitively it seems likely that a continuous equilibrium set would have a stronger and wider region of attraction than an isolated equilibrium point and that variation in parameters or the initial condition will have reduced effect on the performance. Further a method to develop Lyapunov-like functions and to use the nature of the flow to prove stability is shown.

From a biological perspective, this work developed an elementary mathematical reasoning into the causes for the mammalian emotional network to be stable. This reasoning can be considered as good as the original model is, and shall inherit the shortcomings of the original computational model of the limbic system. The original computational model was not intended to be a generic model for all cases; hence this reasoning will not be valid for those cases.

A comparison with established adaptive control algorithms which are also error-driven is not presented here. Some comparisons with other established control approaches are reported in [12], [13]; however continuous time systems are not covered. 
Further developments of this algorithm can be focused on two lines: attempting higher order and MIMO systems and attempting several amygdala and orbitofrontal cortex loops.

Higher order plants would present a challenge in finding the first integrals as the Frobenius method is fairly involved for the present case itself. In fact the Frobenius method is only limited to 3-dimensional problems: hence extensions or other algorithms need to be searched for. Few cases of nonlinear plants were attempted, but the elegance of the method for choosing positively invariant sets was diminished as one of the nullclines is no longer a plane.

Having multiple loops raises a more basic issue than the analytical complexity. A biological situation is far more complex than an engineering problem, and it is clear that there are multiple sensory measurements that need to be taken into account for an appropriate decision. However for engineering goals, we shall have to identify methods so that each loop of the amygdala and the orbitofrontal cortex would be different from others: either have multiple measurements or have different weights for the same input to develop different emotional signals. 


\section{REFERENCES}

[1] J. Martínez-Miranda and A. Aldea, "Emotions in human and artificial intelligence", Computers in Human Behavior, vol. 21, pp. 323-341, 2005.

[2] J. E. LeDoux, The Emotional Brain. New York, NY: Simon \& Schuster, 1996.

[3] A. M. Meystel and J. S. Albus, Intelligent Systems: Architecture, Design and Control. New York: Wiley Interscience, 2002.

[4] J. S. Albus, "Outline for a theory of intelligence", IEEE Transactions on Systems, Man and Cybernetics, Vol. 21, No. 3, pp. 473-509, May-June 1991.

[5] T. Samad, "Technologies for engineering autonomy and intelligence", in Proceedings of Performance Metrics for Intelligent Systems (PerMIS), at NIST, Gaithersburg, MD. Aug 14-16, 2000.

[6] M. D. Lewis, I. Granic, (eds.) Emotion, Development, and Self-Organization: Dynamic Systems Approaches to Emotional Development. Cambridge, UK: Cambridge University Press, 2000.

[7] J. S. Barlow, The Cerebellum and Adaptive Control. Cambridge, UK: Cambridge University Press, 2002.

[8] J. Morén, "Emotion and learning- a computational model of the Amygdala", Ph.D. Dissertation, Dept. of Cognitive Science, Lund University, Lund, Sweden, 2002.

[9] P. J. Morgane, D. J. Mokler, “The limbic brain: Continuing resolution”, Neuroscience and Biobehavioral Reviews, (in press), 2005.

[10] D. W. Patterson, L. A. Schmidt, "Neuroanatomy of the human affective system", Brain and Cognition, Vol. 52, pp. 24-26, 2003. 
[11] J. Morén, and C. Balkenius, "A computational model of emotional learning in the Amygdala" in From Animals to Animats 6, Cambridge, MA: MIT Press, 2000, pp. 383-391.

[12] C. Lucas, D. Shahmirzadi, and N. Sheikholeslami, "Introducing BELBIC- Brain Emotional Learning Based Intelligent Controller” International Journal of Intelligent Automation and Soft Computing, vol. 10, no. 1, 2004.

[13] M. Fatourechi, C. Lucas, and A. Khaki-Sedigh, "Reducing control effort by means of emotional learning", in Proc. of $19^{\text {th }}$ Iranian Conference on Electrical Engineering, ICEE 2001, Tehran, Iran, May 2001, pp. 41-1-41-8.

[14] M. W. Hirsch, and S. Smale, Differential Equations, Dynamical Systems, and Linear Algebra. New York: Academic Press, 1974, ch. 12.

[15] T. M. Rocha Filho, I. M. Gléria, A. Figueiredo, and L. Brenig, "The LotkaVolterra canonical format”, Ecological Modelling, vol. 183, pp. 95-106, 2005.

[16] Yu. A. Pykh, "Lyapunov functions for Lotka-Volterra systems: an overview and problems”, in Proc. of $5^{\text {th }}$ IFAC Symposium “Nonlinear Control Systems”, 2001, pp. $1655-1660$.

[17] M. Plank, "On the dynamics of the Lotka-Volterra equations having an invariant hyperplane", SIAM Journal of Applied Mathematics, Vol. 59, No. 5, pp. 1540-1551, 1999.

[18] H. W. Hethcote, "The mathematics of infectious diseases", SIAM Review, Vol. 42, No. 4, pp. 599-653, 2000.

[19] M. Vidyasagar, Nonlinear Systems Analysis. Englewood Cliffs: Prentice-Hall, 1978, ch. 2 . 
[20] Q. Han, L. -Z. Liao, H. Qi, and L. Qi, "Stability analysis of gradient based neural networks for optimization problems", Journal of Global Optimization, vol. 19, pp. 363-381, 2001.

[21] H. K. Khalil, Nonlinear Systems. Upper Saddle River, NJ: Prentice Hall, $3^{\text {rd }}$ ed., 2002, ch. 4 .

[22] A. Goriely, Integrability and Nonintegrability of Dynamical Systems, Singapore: World Scientific, 2001, ch. 2.

[23] D. K. Arrowsmith and C. M. Place, Dynamical Systems: Differential Equations, Maps and Chaotic Behaviour. London, UK: Chapman \& Hall, $1^{\text {st }}$ ed., 1992, ch. 3.

[24] N. Rouche, P. Habets and M. LaLoy, Stability Theory by Liapunov's Direct Method. New York, NY: Springer-Verlag, 1977, ch. IV.

[25] M. Fečkan, “A generalization of Bendixson's criterion”, Proc. of the American Mathematical Society, Vol. 129, No. 11, pp. 3395-3399, 2001. 


\section{VITA}

Manik Chandra earned the degree of Bachelor of Technology in mechanical engineering from Indian Institute of Technology Kanpur, Kanpur, India in 2003. Thereafter he joined the Department of Mechanical Engineering at Texas A\&M University, College Station for graduate studies and earned a Master of Science degree in December 2005. He is interested in nonlinear control theory and differential equations.

Manik Chandra can be reached at the following address:

Manik Chandra

110/195 Ram Krishna Nagar

Kanpur, 208012

INDIA

Email: manikchandra@yahoo.com 